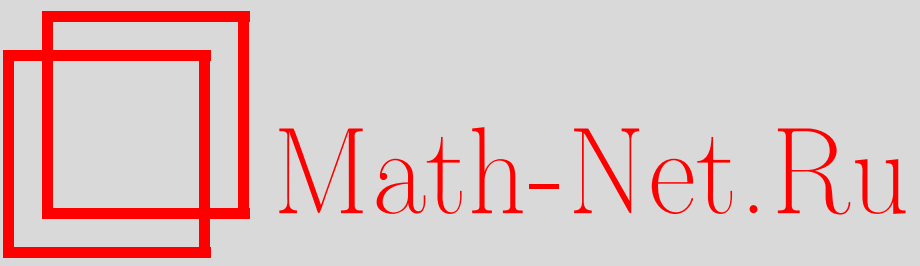

А. С. Холево, Квантовые теоремы кодирования, УМН, 1998, том 53, выпуск $6,193-230$

DOI: https://doi.org/10.4213/rm91

Использование Общероссийского математического портала Math-Net.Ru подразумевает, что вы прочитали и согласны с пользовательским соглашением

http://www.mathnet.ru/rus/agreement

Параметры загрузки:

IP : 54.174 .149 .18

26 апреля 2023 г., $17: 41: 42$ 


\title{
КВАНТОВЫЕ ТЕОРЕМЫ КОДИРОВАНИЯ
}

\author{
А. С. Холево
}

\section{СОДЕРЖАНИЕ}

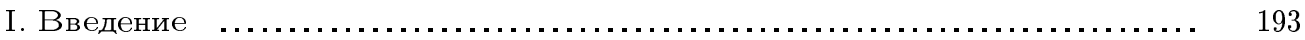

II. Общие рассмотрения

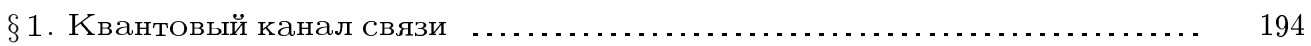

$\S 2$. Энтропийная граница и пропускная способность ................... 197

§3. Формулировка квантовой теоремы кодирования. Слабое обращение 201

III. Доказательство прямого утверждения теоремы кодирования $\ldots . . . .203$

$\S 1$. Каналы с чистыми сигнальными состояниями . ................... 203

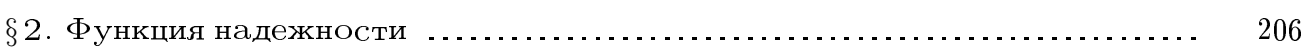

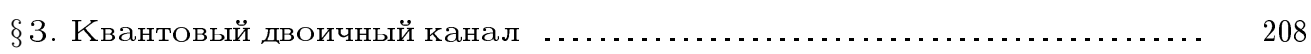

$\S 4$. Случай произвольных состояний с ограниченной энтропией $\ldots . . \ldots . .210$

IV. с-q каналы с ограничениями на входе . . . . . . . . . . . . . . . . . . . . . 213

$\S 1$. Теорема кодирования $\ldots \ldots \ldots \ldots \ldots \ldots \ldots \ldots \ldots \ldots \ldots \ldots \ldots \ldots \ldots \ldots \ldots . . \ldots \ldots \ldots$

$\S 2$. Гауссовский канал с одной степенью свободы $\ldots \ldots \ldots \ldots \ldots \ldots \ldots \ldots \ldots . . \ldots \ldots$

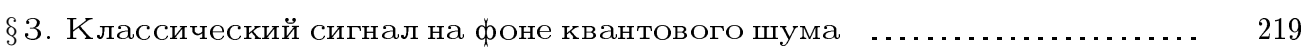

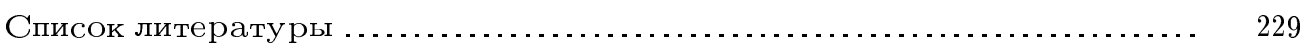

\section{І. Введение}

Вслед за появлением 50 лет назад пионерских работ Шеннона и созданием математических основ теории информации, см., например, [17], встал вопрос о фундаментальных ограничениях на возможности передачи сообшений, накладьваемых природой физического носителя информации. Проблема определения пропускной способности квантового канала связи сфформировалась в 60-е годы (см., например, [13], [10], [34], [14], а также обзор [7]) и восходит к более ранним классическим трудам Габора и Бриллюэна, поставившим вопрос о квантово-механических пределах точности

Работа выполнена при частичной поддержке Российского фонда фундаментальных исследований (гранты № № 96-01-01709, 96-15-96033). 
и скорости передачи информации. Эти работы заложили физические основы и подняли проблему адекватного математического рассмотрения всего данного круга вопросов. Принципиальные шаги в этом направлении были сделаны в 70-е годы, когда была построена некоммутативная теория статистических решений [16], [21], найдено доказательство квантовой энтропийной границы [19] и обнаружена строгая супераддитивность шенноновской информации в квантовом канале связи без памяти [24].

Существенный прогресс был достигнут в последние годы, когда были доказаны прямые теоремы кодирования, устанавливаюшие достижимость квантовой энтропийной границы [15], [26], [42]. В определенной мере этот прогресс стимулирован современньм развитием идей квантовой теории информации и квантового компьютинга (см., например, [4]). Вопрос о пропускной способности квантового канала связи представляет большой интерес в связи с недавним открытием квантовых кодов, исправляющих ошибки $[6],[43]$. Здесь следует отметить, что различают классическую и квантовую пропускные способности квантового канала связи. Установившегося определения квантовой пропускной способности, которая связана с асимптотически безошибочной передачей квантовых состояний и представляет основной интерес в квантовом компютинге, пока нет. Важные шаги в этом направлении сделаны, в частности, в работах [2], [1], где предложена некоторая верхняя гранища, однако вопрос о достижимости этой гранищы остается открытьм.

В настояшей статье дается математически строгое и замкнутое изложение круга вопросов, связанных с классической пропускной способностью квантового канала, определяемой как максимальное количество шенноновской информации, которое может быть асимптотически безошибочно передано по квантовому каналу при произвольном блочном кодировании. Эта тема представляет самостоятельный интерес и в то же время является необходимым звеном при переходе от классической к чисто квантовой теории информации. Основное внимание уделено последним достижениям; кратко обозревается также история вопроса, сформулирован ряд нерешенных задач.

\section{II. Общие рассмотрения}

\section{§ 1. Квантовый канал связи}

С математической точки зрения канал связи в общем случае представляет собой аффинное отображение, которое переводит состояния на входе канала в состояния на выходе. Состояния представляют статистические ансамбли, которые могут подвергаться смешиванию, и аффинность выражает фундаментальное свойство сохранения статистических смесей. В классическом случае состояния описываются распределениями вероятностей, и классический канал связи задается переходным отображением распределений на входе в распределения на выходе. Если же по меньшей мере одна из систем, описывающих вход или выход, является квантовой, то говорят о квантовом канале связи.

Пусть $\mathscr{H}$ - сепарабельное гильбертово пространство, которое дает математическое описание физического носителя информации. Мы не предполагаем $\mathscr{H}$ конечномерным, как это делается обычно в задачах квантового компютинга, так как такое предположение не вьполняется, например, для гауссовского канала (см. §IV.3). 
В данном контексте квантовое состояние задается оператором плотности, т.е. положительньм оператором $S$ в $\mathscr{H}$ с единичньм следом, $\operatorname{Tr} S=1$. Используя формализм Дирака, мы будем обозначать векторы пространства $\mathscr{H}$ через $|\psi\rangle$, а эрмитово сопряженные векторы дуального пространства через $\langle\psi|$. Тогда $\langle\phi \mid \psi\rangle$ обозначает скалярное (внутреннее) произведение векторов $|\phi\rangle,|\psi\rangle$, а $|\psi\rangle\langle\phi|$ - внешнее произведение, т.е. оператор $A$ ранга 1, действуюший на вектор $|\chi\rangle$ как $A|\chi\rangle=|\psi\rangle\langle\phi \mid \chi\rangle$. Если $|\psi\rangle$ единичньй вектор, то $|\psi\rangle\langle\psi|$ является ортогональным проектором на $|\psi\rangle$. Это частный случай оператора плотности, представляюшего иистое состояние системы. Чистые состояния являются крайними точками вьпуклого множества $\mathscr{S}(\mathscr{H})$ всех состояний; произвольное квантовое состояние $S$ представляется в виде смеси чистых состояний. В самом деле, согласно спектральной теореме для вполне непрерьвных операторов,

$$
S=\sum_{i} \lambda_{i}\left|\psi_{i}\right\rangle\left\langle\psi_{i}\right|
$$

где $\lambda_{i}$ - собственные числа, $\left|\psi_{i}\right\rangle$ - собственные векторы оператора $S$ (ряд сходится по ядерной норме). Отметим, что $\left\{\lambda_{i}\right\}$ образуют распределение вероятностей, т.е. классическое состояние на спектре оператора $S$. С другой стороны, всякое (дискретное) классическое состояние аффинно вкладьвается таким образом в $\mathscr{S}(\mathscr{H})$, если зафиксировать ортонормированньй базис $\left\{\left|\psi_{i}\right\rangle\right\}$ в $\mathscr{H}$.

Следующее важное понятие квантового решающего правила является далеко идущим обобщением стандартного определения квантовой наблюдаемой. Математически оно описьвается разложением единищы в гильбертовом пространстве $\mathscr{H}$, т.е. семейством $X=\left\{X_{j}\right\}$ положительных операторов в $\mathscr{H}$, удовлетворяющим условию $\sum_{j} X_{j}=I$, где $I$ - единичный оператор в $\mathscr{H}$. Стандартное определение наблюдаемой получается, если дополнительно потребовать, что $X_{j}$ являются взаимно ортогональными проекторами, $X_{j} X_{k}=\delta_{j k} X_{j}$. Знаменитая теорема М. А. Наймарка [39] утверждает, что всякое разложение единицы может быть расширено до ортогонального в некотором объемлющем гильбертовом пространстве. С физической точки зрения это означает, что всякое решающее правило эквивалентно измерению наблюдаемой в некотором расширении исходной квантовой системы [25]. Вероятность принятия решения $j$ для решаюшего правила $X$, примененного к системе в состоянии $S$, по определению полагается равной

$$
P(j \mid S)=\operatorname{Tr} S X_{j}
$$

Это соотношение переходит в статистический постулат Борна в случае обычной наблюдаемой и, в свою очередь, эквивалентно формуле Борна для наблюдаемой расширенной системы, реализуюшей решаюшее правило $X$.

Система векторов $\left\{\left|\phi_{j}\right\rangle\right\}$ в $\mathscr{H}$ назьвается переполненной, если $\sum_{j}\left|\phi_{j}\right\rangle\left\langle\phi_{j}\right|=I$. Всякая переполненная система, в частности, всякий ортонормированньй базис порождает решаюшее правило $X$, для которого $X_{j}=\left|\phi_{j}\right\rangle\left\langle\phi_{j}\right|$ и $P(j \mid S)=\left\langle\phi_{j} \mid S \phi_{j}\right\rangle$.

Отображение $S \rightarrow P(\cdot \mid S)$ является аффинным, и обратно, всякое аффинное отображение множества квантовых состояний в распределения вероятностей имеет такой вид (см. [25, предложение 1.6.1]). Фактически, такое отображение уже дает пример квантового канала (назьваемого q-с каналом, см. (19)). 
Классический случай вписьвается в эту картину, если рассматриваемые операторы коммутируют между собой и, следовательно, диагональны в некотором базисе, индексированном переменной $\omega$; в самом деле, полагая $S=\operatorname{diag}[S(\omega)]$, $X_{j}=\operatorname{diag}[X(j \mid \omega)]$, где $S(\omega)$ - классическое состояние, а $X(j \mid \omega)$ - классическое решаюшее правило, мы получаем классическую формулу $P(j \mid S)=\sum_{\omega} X(j \mid \omega) S(\omega)$. Квантовые наблюдаемые соответствуют при этом классическим детерминированньм решаюшим правилам (т.е. случайным величинам).

В ранних работах (см., например, [9]) квантовьй канал связи определялся как произвольное аффинное отображение $\Phi$ множества состояний $\mathscr{S}(\mathscr{H})$. Нетрудно доказать, что всякое такое отображение однозначно продолжается до положительного линейного отображения банахова пространства ядерных операторов в $\mathscr{H}$, сохраняющего след. Позднее стало ясно, что свойство положительности в этом определении должно быть сушественно усилено [18], [32], [38]. Линейное отображение $\Phi$ называется вполне положительным, если для любого конечного набора векторов $\left\{\left|\phi_{i}\right\rangle,\left|\psi_{i}\right\rangle\right\} \subset \mathscr{H}$ вьполняется

$$
\sum_{i, j}\left\langle\phi_{i}\left|\Phi\left[\left|\psi_{i}\right\rangle\left\langle\psi_{j}\right|\right]\right| \phi_{j}\right\rangle \geqslant 0
$$

(это только одно из целого ряда эквивалентных определений). Известньй результат Стайнспринга [44] (обобщаюший упомянутую вьше теорему М. А. Наймарка) утверждает, что вполне положительное отображение расширяется до представления алгебры (в нашем случае, алгебры всех ограниченных операторов в $\mathscr{H}$ ) в некотором объемлющем пространстве. В квантовой физике это равносильно реализуемости отображения посредством унитарной эволюции некоторой объемлющей системы, включающей окружение исходной системы [32], [38]. Основьваясь на теореме Стайнспринга, нетрудно показать, что всякое вполне положительное отображение, сохраняющее след и непрерьвное в слабой операторной топологии, имеет вид

$$
\Phi[S]=\sum_{k} V_{k} S V_{k}^{*}
$$

где $V_{k}$ - ограниченные операторы такие, что $\sum_{k} V_{k}^{*} V_{k}=I$. В дальнейшем мы называем такое отображение каналом. ${ }^{1}$

Рассмотрим два важных частных случая. Пусть $\left\{S_{i}\right\}$ - семейство квантовых состояний и $\left\{X_{i}\right\}-$ разложение единищы в $\mathscr{H}$. Положим

$$
\Phi[S]=\sum_{i} S_{i} \operatorname{Tr} S X_{i}
$$

Легко проверяется, что это отображение является вполне положительным и сохраняющим след. Если $X_{i}=\left|e_{i}\right\rangle\left\langle e_{i}\right|$, где $\left\{e_{i}\right\}$ - ортонормированньй базис, мы назьваем

\footnotetext{
${ }^{1}$ В недавней статье [11] рассматривается более общее определение, не предполагающее полной положительности. Такие попытки могут представлять интерес в свете недавнего наблюдения [31], что положительные, но не вполне положительные отображения возникают в моделях взаимодействия с более сложным окружением (таким, как неабелево калибровочное поле), описьваемых операторами в градуированном, а не обычном тензорном произведении гильбертовых пространств.
} 
его $c-q($ кассически-квантовым) каналом. Оно однозначно задается отображением $i \rightarrow S_{i}$ входного алфавита $A=\{i\}$ в сигнальные квантовые состояния $S_{i}$. Если при этом операторы $S_{i}$ коммутируют, то канал называется квазиклассическим; такой канал эквивалентен классическому каналу с переходньми вероятностями, задаваемьми собственньми числами $S(\omega \mid i)$ операторов $S_{i}$.

С другой стороны, если $X_{i}$ - произвольное разложение единицы, а $S_{i}=\left|e_{i}\right\rangle\left\langle e_{i}\right|$, мы называем канал $q-c$ (квантово-классическим) каналом, поскольку он задается решаюшим правилом, отображаюшим квантовые состояния в распределения вероятностей на выходном алфавите $B=\{i\}$. Каналы вида (3), разумеется, не исчерпьвают всех возможностей; простейший пример, не сводяшийся к (3), дается обратимой эволюцией

$$
\Phi[S]=V S V^{*},
$$

где $V$ - произвольньй унитарньй оператор.

\section{§. Энтропийная граница и пропускная способность}

Пусть $\pi$ - дискретное распределение вероятностей на $\mathscr{S}(\mathscr{H})$, приписываюшее вероятность $\pi_{i}$ состоянию $S_{i}$. Мы обозначаем

$$
\Delta H(\pi)=\sum_{i} \pi_{i} H\left(S_{i} ; \bar{S}_{\pi}\right),
$$

где

$$
\bar{S}_{\pi}=\sum_{i} \pi_{i} S_{i},
$$

a

$$
H\left(S ; S^{\prime}\right)=\operatorname{Tr} S\left(\log S-\log S^{\prime}\right)
$$

- квантовая относительная энтропия (более точное определение и обсуждение свойств см. в $[38],[47],[40])$. Как и относительная энтропия, величина $\Delta H(\pi)$ неотрицательна, но может быть бесконечной. Если

$$
\sup _{i} H\left(S_{i}\right)<\infty
$$

где $H(S)=-\operatorname{Tr} S \log S-$ квантовая энтропия фон Неймана, то

$$
\Delta H(\pi)=H\left(\bar{S}_{\pi}\right)-\bar{H}\left(S_{(\cdot)}\right),
$$

где $\bar{H}\left(S_{(\cdot)}\right)=\sum_{i} \pi_{i} H\left(S_{i}\right)<\infty$.

Пусть $X=\left\{X_{j}\right\}$ - решаюшее правило и $P(j \mid i)=\operatorname{Tr} S_{i} X_{j}$ - соответствуюшие переходные вероятности. Обозначим

$$
I(\pi, X)=\sum_{j} \sum_{i} \pi_{i} P(j \mid i) \log \left(\frac{P(j \mid i)}{\sum_{k} \pi_{k} P(j \mid k)}\right)
$$


классическое шенноновское количество информации между случайными величинами, описьваюшими вход и выход канала связи. Имеет место квантовая энтропийная граница

$$
\sup _{X} I(\pi, X) \leqslant \Delta H(\pi)
$$

причем равенство достигается тогда и только тогда, когда операторы $\pi_{i} S_{i}$ коммутируют. В виде предположения это неравенство было впервые сформулировано в работе [14] в контексте стандартной квантовой теории измерения. Доказательство в конечномерном случае, основанное на исследовании свойств выпуклости правой и левой частей в (10), было дано в [19].

Другой подход к доказательству этого неравенства может быть основан на "обобщенной Н-теореме", установленной позднее в серии работ [38] и в [46], в основе которой лежит свойство сильной субаддитивности квантовой энтропии [37]. Именно, для любых состояний $S, S^{\prime}$ и канала $\Phi$ имеет место неравенство

$$
H\left(\Phi(S) ; \Phi\left(S^{\prime}\right)\right) \leqslant H\left(S ; S^{\prime}\right) .
$$

Оно может быть использовано для доказательства энтропийной границы в полной обшности, если в качестве $\Phi$ взять q-с канал, соответствуюший решаюшему правилу $X$ (см. [48]). Первоначальное доказательство, данное в [19], также переносится на бесконечномерный случай при выполнении условия (7), однако переформулировка энтропийной границы в терминах условной энтропии позволяет охватить и тот случай, когда это условие не выполняется и соотношение (8) не имеет места (в частности, состояния $S_{i}$ могут иметь бесконечную энтропию).

Если $\Phi$ некоторьй канал, то мы обозначаем $I(\pi, \Phi, X)$ количество информации, определяемое аналогично (9), но с переходными вероятностями $P(j \mid i)=\operatorname{Tr} \Phi\left[S_{i}\right] X_{j}$, и

$$
\Delta H(\pi, \Phi)=\sum_{i} \pi_{i} H\left(\Phi\left[S_{i}\right] ; \Phi\left[\bar{S}_{\pi}\right]\right)
$$

Имея в виду рассмотрение блочного кодирования, введем тензорную степень канала $\Phi^{\otimes n}=\Phi \otimes \cdots \otimes \Phi$ в гильбертовом пространстве $\mathscr{H}^{\otimes n}=\mathscr{H} \otimes \cdots \otimes \mathscr{H}$. Обозначим

$$
C_{n}(\Phi)=\sup _{\pi} \sup _{X} I\left(\pi, \Phi^{\otimes n}, X\right) ; \quad \bar{C}_{n}(\Phi)=\sup _{\pi} \Delta H\left(\pi, \Phi^{\otimes n}\right),
$$

где супремумы берутся по всевозможным дискретным распределениям вероятностей $\pi$ на $\mathscr{S}\left(\mathscr{H}^{\otimes n}\right)$ и по всевозможньм решающим правилам $X$ в $\mathscr{H}^{\otimes n}$. Легко видеть, что последовательности $C_{n}(\Phi), \bar{C}_{n}(\Phi)$ обладают свойством супераддитивности:

$$
C_{n}(\Phi)+C_{m}(\Phi) \leqslant C_{n+m}(\Phi), \quad \bar{C}_{n}(\Phi)+\bar{C}_{m}(\Phi) \leqslant \bar{C}_{n+m}(\Phi) .
$$

Отсюда вытекает существование пределов

$$
\begin{aligned}
& C(\Phi)=\lim _{n \rightarrow \infty} \frac{C_{n}(\Phi)}{n}=\sup _{n} \frac{C_{n}(\Phi)}{n}, \\
& \bar{C}(\Phi)=\lim _{n \rightarrow \infty} \frac{\bar{C}_{n}(\Phi)}{n}=\sup _{n} \frac{\bar{C}_{n}(\Phi)}{n} .
\end{aligned}
$$


Из энтропийной границы получаем

$$
C(\Phi) \leqslant \bar{C}(\Phi)
$$

Для (квази)классического канала последовательность $C_{n}(\Phi)$ аддитивна, $C_{n}(\Phi)=$ $n C_{1}(\Phi)$, и, очевидным образом, $C(\Phi)=\bar{C}(\Phi)=C_{1}(\Phi)$. Одно из радикальных и наиболее важных отличий квантового случая состоит в возможности неравенства $C_{1}(\Phi)<C(\Phi)$, влекушего строгую супераддитивность последовательности $C_{n}(\Phi)$. Парадоксальным образом, квантовые каналы, которые являются естественным обобщением классических каналов без памяти, обладают специфической "квантовой памятью”. Этот феномен можно интерпретировать как двойственное проявление известных “коррелящий Эйнштейна-Подольского-Розена" в квантовых измерениях над составньми системами (более подробно см. в [26]).

Мы назьваем величину $C(\Phi)$ пропускной способностью квантового канала связи. Мотивацию такого определения можно получить, основьваясь на классической теореме кодирования Шеннона (cp. [24]), но мы дадим другое обоснование, из которого вытекает также значительно более глубокий факт

$$
C(\Phi)=\bar{C}(\Phi)
$$

В некоторых случаях (см. ниже) последовательность $\bar{C}_{n}(\Phi)$ оказьвается аддитивной, что позволяет избавиться от предела в (14) и дает принципиальную возможность выгислить пропускную способность по формуле

$$
\bar{C}(\Phi)=\bar{C}_{1}(\Phi)=\sup _{\pi} \Delta H(\pi, \Phi)
$$

В работе [3] был поставлен вопрос об аддитивности этой последовательности, которьй в обшем случае до сих пор остается не разрешенньм. Аддитивность тривиальньм образом имеет место для обратимых каналов (4). Приведем частичный результат в этом направлении.

ПРЕДЛОЖЕНИЕ 1. Если $\Phi$ есть $c-q$ или $q-c$ канал, то

$$
\bar{C}_{n}(\Phi)+\bar{C}_{m}(\Phi)=\bar{C}_{n+m}(\Phi) .
$$

ДокАЗАТЕльство. Достаточно показать, что

$$
\bar{C}_{1}(\Phi)+\bar{C}_{1}(\Phi) \geqslant \bar{C}_{2}(\Phi) .
$$

Если $\Phi$ является с-q каналом, т.е.

$$
\Phi[S]=\sum_{i} S_{i}\left\langle e_{i}|S| e_{i}\right\rangle
$$

где $S_{i}-$ фиксированные состояния в $\mathscr{H}$, то

$$
\sup _{\pi} \Delta H(\pi, \Phi)=\sup _{\pi_{i}} \Delta H(\pi)
$$


где $\Delta H(\pi)$ дается выражением (5) с этими состояниями $S_{i}$. Рассмотрим распределение $\pi$, которое приписьвает вероятности $\pi_{i j}$ состояниям $S_{i} \otimes S_{j}$ в $\mathscr{H} \otimes \mathscr{H}$. Тогда

$$
\Delta H(\pi) \leqslant \Delta H\left(\pi^{1}\right)+\Delta H\left(\pi^{2}\right),
$$

где $\pi^{1}$ - первое маргинальное распределение, приписывающее вероятности $\pi_{i}^{1}=$ $\sum_{j} \pi_{i j}$ состояниям $S_{i}$, и, аналогично, $\pi^{2}$ - второе маргинальное распределение, приписьваюшее вероятности $\pi_{j}^{2}=\sum_{i} \pi_{i j}$ состояниям $S_{j}$. В конечномерном случае, когда соотношение (8) вьполняется автоматически, это следует из свойства субаддитивности энтропии относительно тензорного произведения (см. доказательство леммы в приложении работы $[26])$. В бесконечномерном случае рассмотрим монотонно возрастающую последовательность ортогональных проекторов $P_{r} \uparrow I$ в $\mathscr{H}$, и введем обозначение

$$
\Delta H_{r}(\pi)=\sum_{i} \pi_{i} H\left(P_{r} S_{i} P_{r} ; P_{r} \bar{S}_{\pi} P_{r}\right) .
$$

Используя свойства относительной энтропии [38], получаем $\Delta H_{r}(\pi) \uparrow \Delta H(\pi)$. Используя соотношение (18) для нормированных спроецированных состояний, имеем

$$
\Delta_{r} H(\pi) \leqslant \Delta H_{r}\left(\pi^{1}\right)+\Delta H_{r}\left(\pi^{2}\right)-\phi\left(\operatorname{Tr}\left(P_{r} \otimes P_{r}\right) \bar{S}_{\pi}\left(P_{r} \otimes P_{r}\right)\right),
$$

где $\phi(x)=-x \log x$. Переходя к пределу при $r \rightarrow \infty$, получаем неравенство (18) в общем случае. Беря в (18) супремум по $\pi$, получаем (16).

Пусть теперь $\Phi$ является q-с каналом

$$
\Phi[S]=\sum_{j} \operatorname{Tr} S X_{j}\left|e_{j}\right\rangle\left\langle e_{j}\right|,
$$

и пусть $\pi$ - дискретное распределение вероятностей на $\mathscr{S}(\mathscr{H})$, приписьвающее вероятности $\pi_{k}$ состояниям $S_{k}$, тогда операторы плотности $\Phi\left[S_{k}\right]$ коммутируют и величина

$$
\Delta H(\pi, \Phi)=I(\mathscr{K} ; \mathscr{J})
$$

является классическим количеством информации (9), отвечаюшим распределению на входе $\pi$ и переходным вероятностям $P(j \mid k)=\operatorname{Tr} S_{k} X_{j}$. Здесь через $\mathscr{K}$ обозначена входная переменная, принимаюшая значения $k$, а через $\mathscr{J}$-выходная переменная, принимающая значения $j$. Для доказательства неравенства (16) рассмотрим состояния $S_{k}$ в гильбертовом пространстве $\mathscr{H} \otimes \mathscr{H}$, когда переходная вероятность имеет вид

$$
P\left(j_{1}, j_{2} \mid k\right)=\operatorname{Tr} S_{k}\left(X_{j_{1}} \otimes X_{j_{2}}\right)=P^{1}\left(j_{1} \mid j_{2}, k\right) P^{2}\left(j_{2} \mid k\right),
$$

где

$$
P^{1}\left(j_{1} \mid j_{2}, k\right)=\operatorname{Tr}_{1} S_{j_{2}, k}^{1} X_{j_{1}}, \quad P^{2}\left(j_{2} \mid k\right)=\operatorname{Tr}_{2} S_{k}^{2} X_{j_{2}},
$$

и

$$
S_{k}^{2}=\operatorname{Tr}_{1} S_{k}, \quad S_{j_{2}, k}^{1}=\frac{\operatorname{Tr}_{2} S_{k}\left(I \otimes X_{j_{2}}\right)}{\operatorname{Tr} S_{k}\left(I \otimes X_{j_{2}}\right)} .
$$

Здесь $\operatorname{Tr}_{r}$ обозначает (частичньй) след относительно $r$-го тензорного сомножителя $(r=1,2)$ в $\mathscr{H} \otimes \mathscr{H}$. 
Таким образом,

$$
\Delta H(\pi, \Phi \otimes \Phi)=I\left(\mathscr{K} ; \mathscr{J}_{1} \mathscr{J}_{2}\right)=H\left(\mathscr{J}_{1} \mathscr{J}_{2}\right)-H\left(\mathscr{J}_{1} \mathscr{J}_{2} \mid \mathscr{K}\right),
$$

где $H(\cdot), H(\cdot \mid \cdot)$ обозначают, соответственно, энтропию и условную энтропию классических случайных величин. В силу субаддитивности классической энтропии,

$$
H\left(\mathscr{J}_{1} \mathscr{J}_{2}\right) \leqslant H\left(\mathscr{J}_{1}\right)+H\left(\mathscr{J}_{2}\right)
$$

С другой стороны, соотношение (20) влечет равенство

$$
H\left(\mathscr{J}_{1} \mathscr{J}_{2} \mid \mathscr{K}\right)=H\left(\mathscr{J}_{1} \mid \mathscr{J}_{2} \mathscr{K}\right)+H\left(\mathscr{J}_{2} \mid \mathscr{K}\right) .
$$

Таким образом,

$$
I\left(\mathscr{K} ; \mathscr{J}_{1} \mathscr{J}_{2}\right) \leqslant I\left(\mathscr{K} \mathscr{J}_{2} ; \mathscr{J}_{1}\right)+I\left(\mathscr{K} ; \mathscr{J}_{2}\right),
$$

что равносильно соотношению

$$
\Delta H(\pi, \Phi \otimes \Phi) \leqslant \Delta H\left(\pi^{1}, \Phi\right)+\Delta H\left(\pi^{2}, \Phi\right)
$$

где $\pi^{1}$ - распределение вероятностей на $\mathscr{S}(\mathscr{H})$, приписьвающее вероятности $\pi_{k} P^{2}\left(j_{2} \mid k\right)$ состояниям $S_{j_{2}, k}^{1}$, а $\pi^{2}$ - распределение вероятностей на $\mathscr{S}(\mathscr{H})$, приписываюшее вероятности $\pi_{k}$ состояниям $S_{k}^{2}$. Беря супремум по $\pi$, получаем (16).

\section{§ 3. Формулировка квантовой теоремы кодирования. Слабое обращение}

Будем называть кодом размера $M$ набор $\left(S^{1}, X_{1}\right), \ldots,\left(S^{M}, X_{M}\right)$, где $S^{k}$ - состояния, а $\left\{X_{k}\right\}$ - положительные операторы в $\mathscr{H}^{\otimes n}$, удовлетворяюшие условию $\sum_{k=1}^{M} X_{k} \leqslant I$. Полагая $X_{0}=I-\sum_{k} X_{k}$, имеем разложение единишы в $\mathscr{H}^{\otimes n}$. Значение $k \geqslant 1$ на выходе канала соответствует принятию решения, что было передано состояние $S^{k}$, тогда как значение 0 интерпретируется как уклонение от принятия определенного решения. Средняя вероятность ошибки для такого кода равна

$$
\bar{\lambda}(S, X)=\frac{1}{M} \sum_{k=1}^{M}\left[1-\operatorname{Tr} \Phi\left[S^{k}\right] X_{k}\right]
$$

Обозначим $p(n, M)$ инфимум этой величины по всевозможным кодам размера $M$. С этого момента мы для определенности используем натуральные логарифмы.

Tеорема 1. Eсли $C(\Phi)<\infty u R>C(\Phi)$, mo $p\left(n, \mathrm{e}^{n R}\right) \not \rightarrow$. C другой стороньи, если канал $\Phi$ удовлетворяет условию

$$
\sup _{S \in \mathscr{S}(\mathscr{H})} H(\Phi[S])<\infty
$$

то $p\left(n, \mathrm{e}^{n R}\right) \rightarrow 0$ для $R<\bar{C}(\Phi)$. В частности, $C(\Phi)=\bar{C}(\Phi)$. 
ДокАЗАТЕльство. В основе первого утверждения лежит неравенство

$$
\log M \cdot(1-p(n, M)) \leqslant C_{n}(\Phi)+1
$$

вытекающее из классического неравенства Фано [12], [8]. В самом деле, пусть $\mathscr{J}$ обозначает классическую случайную величину, описьвающую выход канала $\Phi^{\otimes n}$, если используется решаюшее правило $X$, а состояния в коде $(S, X)$ выбираются в соответствии с распределением $\pi_{M}$, приписьваюшим равные вероятности $1 / M$ каждому состоянию $S^{k}$; пусть также $\mathscr{K}$ обозначает случайную величину, значением которой является символ $k$ переданного состояния. Неравенство $Ф$ ано утверждает, что

$$
\log M \cdot(1-\bar{\lambda}(S, X)) \leqslant I(\mathscr{K} ; \mathscr{J})+1 \leqslant C_{n}(\Phi)+1
$$

Полагая $M=\mathrm{e}^{n R}$ и $n \rightarrow \infty$, заключаем, что $p\left(n, \mathrm{e}^{n R}\right) \not \rightarrow 0$ при $R>C$.

Покажем теперь, что доказательство второго утверждения в общем случае сводится к доказательству в случае с-q канала (17), удовлетворяющего условию

$$
\sup _{i} H\left(S_{i}\right)<\infty
$$

Вся следуюшая глава будет посвящена рассмотрению этого случая. Если $R<\bar{C}(\Phi)$, то можно выбрать $n_{0}$ и распределение вероятностей $\pi^{0}$ на $\mathscr{S}\left(\mathscr{H}{ }^{\otimes} n_{0}\right)$ такие, что $n_{0} R<$ $\Delta H\left(\pi^{0}, \Phi^{\otimes n_{0}}\right)$. Пусть $\pi^{0}$ приписьвает вероятность $\pi_{i}^{0}$ состоянию $S_{i}$ в $\mathscr{H}^{\otimes n_{0}} ;$ рассмотрим в этом гильбертовом пространстве с-q канал $\widetilde{\Phi}$, определяемый сотношением

$$
\widetilde{\Phi}[S]=\sum_{i} \Phi^{\otimes n_{0}}\left[S_{i}\right]\left\langle e_{i}|S| e_{i}\right\rangle .
$$

В силу предложения 1 ,

$$
\bar{C}(\widetilde{\Phi})=\sup _{\pi_{i}}\left\{H\left(\sum_{i} \pi_{i} \Phi^{\otimes n_{0}}\left[S_{i}\right]\right)-\sum_{i} \pi_{i} H\left(\Phi^{\otimes n_{0}}\left[S_{i}\right]\right)\right\},
$$

что строго больше, чем $n_{0} R$. Обозначая $\widetilde{p}(n, M)$ инфимум средней вероятности ошибки для канала $\widetilde{\Phi}$, имеем (в предположении, что $n$ кратно $\left.n_{0}\right)$

$$
p\left(n, \mathrm{e}^{n R}\right) \leqslant \widetilde{p}\left(n / n_{0}, \mathrm{e}^{\left(n / n_{0}\right) n_{0} R}\right),
$$

поскольку всякий код размера $M$ для канала $\widetilde{\Phi}$ является кодом того же размера для канала $\Phi$. Отсюда следует, что если утверждение верно для с-q канала $\widetilde{\Phi}$, оно также верно и для исходного канала $\Phi$.

Покажем теперь, что условие $(22)$ влечет $(24)$ для канала $\widetilde{\Phi}$. В самом деле,

$$
\sup _{i} H\left(\Phi^{\otimes n_{0}}\left[S_{i}\right]\right) \leqslant \sup _{S \in \mathscr{S}\left(\mathscr{H} \otimes n_{0}\right)} H\left(\Phi^{\otimes n_{0}}[S]\right) \leqslant n_{0} \sup _{S \in \mathscr{S}(\mathscr{H})} H(\Phi[S])<\infty
$$

в силу субаддитивности квантовой энтропии относительного тензорного произведения. 
С этого момента мы будем рассматривать фиксированньй с-q канал (17) в гильбертовом пространстве $\mathscr{H}$, задаваемый отображением $i \rightarrow S_{i}$ входного алфавита $A=\{i\}$ в множество квантовых состояний $\mathscr{S}(\mathscr{H})$, и уберем $\Phi$ из всех обозначений. Для $n$-й тензорной степени с-q канала слово $w=\left(i_{1}, \ldots, i_{n}\right)$, состоящее из букв входного алфавита, порождает на выходе состояние $S_{w}=S_{i_{1}} \otimes \cdots \otimes S_{i_{n}}$. Код размера $M$ задается набором $\left(w^{1}, X_{1}\right), \ldots,\left(w^{M}, X_{M}\right)$, где $w^{k}$ - слова длины $n$. Средняя вероятность ошибки кода

$$
\bar{\lambda}(W, X)=\frac{1}{M} \sum_{k=1}^{M}\left[1-\operatorname{Tr} S_{w^{k}} X_{k}\right]
$$

В терминах нашего предыдущего определения, это означает, что состояния на входе выбираются в виде

$$
S^{k}=\left|e_{i_{1}}\right\rangle\left\langle e_{i_{1}}|\otimes \cdots \otimes| e_{i_{n}}\right\rangle\left\langle e_{i_{n}}\right|,
$$

где $\left|e_{i}\right\rangle$ берутся из представления (17). Использование произвольных состояний $S^{k}$ сводится к выбору слов кода в соответствии с некоторым распределением вероятностей, что, как известно, не увеличивает скорость передачи.

Доказательство теоремы 1 будет завершено в $\S$ III.2, но сначала мы подробно рассмотрим каналы с чистыми сигнальными состояниями.

\section{III. Доказательство прямого утверждения теоремы кодирования}

\section{§1. Каналы с чистыми сигнальными состояниями}

Рассмотрим с-q канал с чистыми состояниями $S_{i}=\left|\psi_{i}\right\rangle\left\langle\psi_{i}\right|$. Поскольку энтропия чистого состояния равна нулю, условие (7) тривиальньщм образом выполнено и $\Delta H(\pi)=H\left(\bar{S}_{\pi}\right)$. Начиная с обсуждения таких каналов, мы не только следуем истории предмета, но и подготавливаем почву для значительно более сложного случая c-q канала с обшими состояниями. Кроме того, в случае чистых состояний удается получить более глубокие результаты, касаюшиеся асимптотического поведения вероятности ошибки и квантовой функции надежности, которые пока не обобщены на каналы с произвольными состояниями.

Для канала с чистьми состояниями слово $w=\left(i_{1}, \ldots, i_{n}\right)$ на входе порождает вектор состояния $\left|\psi_{w}\right\rangle=\left|\psi_{i_{1}}\right\rangle \otimes \cdots \otimes\left|\psi_{i_{n}}\right\rangle \in \mathscr{H}^{\otimes n}$. Нашей задачей будет получение верхней границы для инфимума $p(n, M)$ вероятности ошибки по всем кодам размера $M$. На первом шаге мы дадим геометрическую оценку сверху для инфимума средней вероятности ошибки

$$
\bar{\lambda}(W, X)=\frac{1}{M} \sum_{k=1}^{M}\left[1-\left\langle\psi_{w^{k}}\left|X_{k}\right| \psi_{w^{k}}\right\rangle\right]
$$

по всевозможным квантовым решаюшим правилам. Минимизация величины (26) является квантовым аналогом байесовской задачи и представляет самостоятельный интерес [20], однако ее аналитическое решение, в отличие от классического случая, не всегда возможно. 
В подпространстве гильбертова пространства $\mathscr{H}^{\otimes n}$, порожденном кодовыми векторами $\left|\psi_{w^{1}}\right\rangle, \ldots,\left|\psi_{w^{M}}\right\rangle$, рассмотрим оператор Грама $G=\sum_{k=1}^{M}\left|\psi_{w^{k}}\right\rangle\left\langle\psi_{w^{k}}\right|$. Этот оператор имеет матрицу $\Gamma=\left[\left\langle\psi_{w^{i}} \mid \psi_{w^{j}}\right\rangle\right]$, относительно переполненной системы

$$
\left|\widehat{\psi}_{w^{k}}\right\rangle=G^{-1 / 2}\left|\psi_{w^{k}}\right\rangle ; \quad k=1, \ldots, M
$$

Отметим, что векторы $\left|\psi_{w^{1}}\right\rangle, \ldots,\left|\psi_{w^{M}}\right\rangle$ могут быть линейно зависимы. В случае линейной независимости, векторы $\left|\widehat{\psi}_{w^{k}}\right\rangle$ образуют ортонормированньй базис, наилучшим образом, в смысле [33], аппроксимируюший систему $\left\{\left|\psi_{w^{k}}\right\rangle\right\}$. Следуя работе[23], введем разложение единицы

$$
X_{k}=\left|\widehat{\psi}_{k}\right\rangle\left\langle\widehat{\psi}_{k}\right|
$$

в рассматриваемом подпространстве. Подставляя решаюшее правило (28) в (26), получаем верхнюю границу

$$
\inf _{X} \bar{\lambda}(W, X) \leqslant \frac{2}{M} \operatorname{Sp}\left(E-\Gamma^{1 / 2}\right)=\frac{1}{M} \operatorname{Sp}\left(E-\Gamma^{1 / 2}\right)^{2},
$$

где $E$ - единичная $(M \times M)$-матрица и $\mathrm{Sp}$ обозначает след $(M \times M)$-матрицы (в отличие от следа оператора). В самом деле, для решаюшего правила (28)

$$
\begin{aligned}
\bar{\lambda}(W, X) & =\frac{1}{M} \sum_{k=1}^{M}\left[1-\left|\left\langle\psi_{w^{k}} \mid \widehat{\psi}_{w^{k}}\right\rangle\right|^{2}\right] \\
& \leqslant \frac{2}{M} \sum_{k=1}^{M}\left[1-\left|\left\langle\psi_{w^{k}} \mid \widehat{\psi}_{w^{k}}\right\rangle\right|\right] \\
& =\frac{2}{M} \sum_{k=1}^{M}\left[1-\left\langle\widehat{\psi}_{w^{k}}\left|G^{\frac{1}{2}}\right| \widehat{\psi}_{w^{k}}\right\rangle\right]
\end{aligned}
$$

что совпадает с (29). При выводе второго соотношения в (29) был использован тот факт, что $\operatorname{Sp} \Gamma=M$ в силу нормированности сигнальных векторов $\left|\psi_{w^{k}}\right\rangle$. Эта граница является точной в том смысле, что имеет место близкая нижняя граница [23]. Однако ее использование осложняется наличием квадратного корня из матрищы Грама. Более простая, но и более грубая граница получается применением неравенства

$$
\left(1-x^{1 / 2}\right)^{2}=(1-x)^{2}\left(1+x^{1 / 2}\right)^{-2} \leqslant(1-x)^{2}, \quad x \geqslant 0,
$$

к собственным числам матрицы $Г$ :

$$
\inf _{X} \bar{\lambda}(W, X) \leqslant \frac{1}{M} \operatorname{Sp}(E-\Gamma)^{2}=\frac{1}{M} \operatorname{Tr} \sum \sum_{k \neq l} S_{w^{k}} S_{w^{l}}
$$

В работе [23] показано, что грубая граница асимптотически эквивалентна более точной границе (29) в пределе “почти ортогональных" состояний, когда $\Gamma \rightarrow E$. 
Чтобы оценить инфимум по всевозможным выборам кодовых слов, применим метод случайного кодирования. Именно, фиксируем распределение $\pi=\left\{\pi_{i}\right\}$ и предположим, что слова $w^{1}, \ldots, w^{M}$ выбираются случайным образом, независимо друг от друга, с одинаковым распределением вероятностей

$$
\mathrm{P}\left\{w=\left(i_{1}, \ldots, i_{n}\right)\right\}=\pi_{1} \cdot \ldots \cdot \pi_{n} .
$$

Тогда математическое ожидание $S_{w}$ равно

$$
\mathrm{E} S_{w}=\sum_{i_{1}, \ldots, i_{n}} \pi_{i_{1}} \cdot \ldots \cdot \pi_{i_{n}}\left|\psi_{i_{1}}\right\rangle\left\langle\psi_{i_{1}}|\otimes \cdots \otimes| \psi_{i_{n}}\right\rangle\left\langle\psi_{i_{n}}\right|=\bar{S}_{\pi}^{\otimes n}
$$

и, усредняя неравенство (31), мы получаем в силу независимости слов $w^{k}, w^{l}$

$$
p(n, M) \leqslant \operatorname{Einf}_{X} \bar{\lambda}(W, X) \leqslant(M-1) \operatorname{Tr}\left(\bar{S}_{\pi}^{\otimes n}\right)^{2}=(M-1) \mathrm{e}^{-n \log \operatorname{Tr} \bar{S}_{\pi}^{2}}
$$

Обозначая

$$
\widetilde{C}=-\log \inf _{\pi} \operatorname{Tr} \bar{S}_{\pi}^{2}=-\log \inf _{\pi} \sum_{i, j} \pi_{i} \pi_{j}\left|\left\langle\psi_{i} \mid \psi_{j}\right\rangle\right|^{2}
$$

мы заключаем,что $C \geqslant \widetilde{C}$. Известны примеры (квантовый двоичньй канал, см. $\S 3$ ниже), в которых $\widetilde{C}>C_{1}$, так что приведенное вьше рассуждение позволяет установить возможность неравенства $C>C_{1}$, из которого вытекает строгая супераддитивность последовательности $C_{n}[24]$. Однако оно недостаточно для доказательства прямой теоремы кодирования, поскольку $\widetilde{C}<\bar{C}$, если только канал не является квазиклассическим. Детальное сравнение величин $C_{1}, \bar{C}$ для различных квантовых каналов связи проведено в работе [30]. Величина $\widetilde{C}$ обсуждалась в работах [24], [45], но ее информационный смысл вполне проясняется лиш в связи с введением квантовой функции надежности (см. ниже соотношение (43)).

Доказательство неравенства $C \geqslant \bar{C}$, данное в работе [15], основьвается на оценках, аналогичных приведенньм вьше, но улучшенных с помошюю проецирования на “типичное подпространство” оператора плотности $\bar{S}_{\pi}^{\otimes n}$. Таким образом, неточность упрощенной гранищы (31) компенсируется устранением “нетипичных" компонент векторов сигнальных состояний. Дадим определение типичного подпространства. Для этого фиксируем малое положительное $\delta$ и обозначим через $\lambda_{j}$ собственные числа, а через $\left|e_{j}\right\rangle$ собственные векторы оператора плотности $\bar{S}_{\pi}$. Тогда оператор $\bar{S}_{\pi}^{\otimes n}$ имеет собственные числа $\lambda_{J}=\lambda_{j_{1}} \cdot \ldots \cdot \lambda_{j_{n}}$ и собственные векторы $\left|e_{J}\right\rangle=\left|e_{j_{1}}\right\rangle \otimes \cdots \otimes\left|e_{j_{n}}\right\rangle$, где $J=\left(j_{1}, \ldots, j_{n}\right)$. Проектор на типичное подпространство определяется как

$$
P=\sum_{J \in B}\left|e_{J}\right\rangle\left\langle e_{J}\right|
$$

где $B=\left\{J: \mathrm{e}^{-n\left[H\left(\bar{S}_{\pi}\right)+\delta\right]}<\lambda_{J}<\mathrm{e}^{-n\left[H\left(\bar{S}_{\pi}\right)-\delta\right]}\right\}$. Это понятие играет центральную роль в квантовом аналоге “сжатия данных" при кодировании источника квантовой 
информации [29]. В чисто математическом контексте оно появилось в работе [40, теорема 1.18]. Далее используются следующие его основные свойства: во-первых, из определения,

$$
\left\|\bar{S}_{\pi}^{\otimes n} P\right\|<\mathrm{e}^{-n\left[H\left(\bar{S}_{\pi}\right)-\delta\right]} .
$$

Во-вторых, для фиксированного малого положительного $\epsilon$ и достаточно больших $n$

$$
\operatorname{Tr} \bar{S}_{\pi}^{\otimes n}(I-P) \leqslant \epsilon,
$$

поскольку последовательность $J \in B$ является типичной для распределения вероятностей $\lambda_{J}$ в смысле классической теории информации [12], [8].

Вкратце, идея работы [15] состоит в следующем. Векторы сигнальных состояний $\left|\psi_{w^{k}}\right\rangle$ заменяются ненормированными векторами $\left|\widetilde{\psi}_{w^{k}}\right\rangle=P\left|\psi_{w^{k}}\right\rangle$. Обозначая $\widetilde{\Gamma}$ их матрицу Грама, имеем аналогично неравенству в (29)

$$
\inf _{X} \bar{\lambda}(W, X) \leqslant \frac{2}{M} \operatorname{Sp}\left(E-\widetilde{\Gamma}^{1 / 2}\right) .
$$

Используя вытекающее из (30) неравенство

$$
2-2 x^{1 / 2}=(1-x)+\left(1-x^{1 / 2}\right)^{2} \leqslant(1-x)+(1-x)^{2},
$$

справедливое для $x \geqslant 0$, можно получить

$$
\begin{aligned}
\inf _{X} \bar{\lambda}(W, X) & \leqslant \frac{1}{M}\left\{\operatorname{Sp}(E-\widetilde{\Gamma})+\operatorname{Sp}(E-\widetilde{\Gamma})^{2}\right\} \\
& \leqslant \frac{1}{M} \sum_{k}\left\{2 \operatorname{Tr} S_{w^{k}}(I-P)+\sum_{k \neq l} \operatorname{Tr} S_{w^{k}} P S_{w^{l}} P\right\} .
\end{aligned}
$$

Применяя случайное кодирование и используя (33) и свойства $(35),(36)$ типичного подпространства, получаем для больших $n$

$$
p(n, M) \leqslant 2 \operatorname{Tr} \bar{S}_{\pi}^{\otimes n}(I-P)+(M-1) \operatorname{Tr}\left(\bar{S}_{\pi}^{\otimes n} P\right)^{2} \leqslant 2 \epsilon+(M-1) \mathrm{e}^{-n\left[H\left(\bar{S}_{\pi}\right)-\delta\right]},
$$

откуда вытекает, что $p\left(n, \mathrm{e}^{n R}\right) \rightarrow 0$ при $R<\bar{C}-\delta$, и, следовательно, $C \geqslant \bar{C}$.

\section{§. Функция надежности}

Использование типичных подпоследовательностей упрощает доказательство теорем кодирования в классической теории информации, однако не является необходимым; прямые доказательства, опирающиеся на более точные оценки вероятности ошибки, позволяют также оценить показатель экспоненциальной скорости сходимости вероятности ошибки, так называемую функцию надежности канала [12]

$$
E(R)=\lim _{n \rightarrow \infty} \sup \frac{1}{n} \log \frac{1}{p\left(n, \mathrm{e}^{n R}\right)}, \quad 0<R<C .
$$

Это наводит на мысль попытаться получить аналогичные оценки в квантовом случае.

Teоpema 2. Для всеx $M, n u 0 \leqslant s \leqslant 1$

$$
\mathrm{E} \inf _{X} \bar{\lambda}(W, X) \leqslant 2(M-1)^{s}\left[\operatorname{Tr} \bar{S}_{\pi}^{1+s}\right]^{n} .
$$


ДокАЗАТЕЛЬСТво. Заметим, что

$$
\frac{2}{M} \operatorname{Sp}\left(E-\Gamma^{1 / 2}\right)=\frac{2}{M}\left(M-\operatorname{Tr} G^{1 / 2}\right) .
$$

Рассмотрим два операторных неравенства

$$
\begin{aligned}
& -2 G^{1 / 2} \leqslant-2 G+2 G, \\
& -2 G^{1 / 2} \leqslant-2 G+\left(G^{2}-G\right) .
\end{aligned}
$$

Первое очевидно, а второе следует из (37). Беря математическое ожидание относительно распределения вероятностей (32), получаем

$$
-2 \mathrm{E} G^{1 / 2} \leqslant-2 \mathrm{E} G+\left\{\begin{array}{l}
2 \mathrm{E} G \\
\mathrm{E}\left(G^{2}-G\right)
\end{array} .\right.
$$

Используя (33), имеем

$$
\begin{aligned}
\mathrm{E} G & =\mathrm{E} \sum_{k=1}^{M}\left|\psi_{u^{k}}\right\rangle\left\langle\psi_{u^{k}}|=M \mathrm{E}| \psi_{u^{k}}\right\rangle\left\langle\psi_{u^{k}}\right|=M \bar{S}_{\pi}^{\otimes n}, \\
\mathrm{E}\left(G^{2}-G\right) & =\mathrm{E} \sum_{k=1}^{M} \sum_{l=1}^{M}\left|\psi_{u^{k}}\right\rangle\left\langle\psi_{u^{k}}|| \psi_{u^{l}}\right\rangle\left\langle\psi_{u^{l}}\left|-\mathrm{E} \sum_{k=1}^{M}\right| \psi_{u^{k}}\right\rangle\left\langle\psi_{u^{k}}\right| \\
& =\mathrm{E} \sum_{k \neq l}\left|\psi_{u^{k}}\right\rangle\left\langle\psi_{u^{k}} \mid \psi_{u^{l}}\right\rangle\left\langle\psi_{u^{l}}\right|=M(M-1)\left[\bar{S}_{\pi}^{\otimes n}\right]^{2} .
\end{aligned}
$$

Пусть $\left\{e_{J}\right\}$ - ортонормированный базис из собственных векторов, $\lambda_{J}$ - соответствуюшие собственные значения оператора $\bar{S}_{\pi}^{\otimes n}$. Тогда

$$
-2\left\langle e_{J}\left|\mathrm{E} G^{1 / 2}\right| e_{J}\right\rangle \leqslant-2 M \lambda_{J}+M \lambda_{J} \min \left(2,(M-1) \lambda_{J}\right) .
$$

Используя неравенство $\min \{a, b\} \leqslant a^{s} b^{1-s}, 0 \leqslant s \leqslant 1$, получаем

$$
\min \left(2,(M-1) \lambda_{J}\right) \leqslant 2(M-1)^{s} \lambda_{J}^{s}, \quad 0 \leqslant s \leqslant 1 .
$$

Суммируя по $J$ и деля на $M$, получаем из (29), (40)

$$
\operatorname{Einf}_{X} \bar{\lambda}(W, X) \leqslant 2(M-1)^{s} \sum_{J} \lambda_{J}^{1+s}=2(M-1)^{s}\left[\operatorname{Tr} \bar{S}_{\pi}^{1+s}\right]^{n}, \quad 0 \leqslant s \leqslant 1 .
$$

Вводя потенциал $\mu(\pi, s)$, подобно тому, как это делается в классической теории информации $[12$, гл. 5],

$$
\mu(\pi, s)=-\log \operatorname{Tr} \bar{S}_{\pi}^{1+s},
$$

и полагая $M=\mathrm{e}^{n R}$, получаем

$$
E(R) \geqslant \sup _{0 \leqslant s \leqslant 1}\left(\sup _{\pi} \mu(\pi, s)-s R\right) \equiv E_{r}(R) .
$$


Отсюда, в частности, следует утверждение прямой теоремы кодирования

$$
C \geqslant \sup _{\pi} \mu^{\prime}(\pi, 0)=\bar{C} .
$$

С другой стороны, оказьвается возможным модифицировать классический “метод выбрасывания" [12, гл. 5.7], что приводит к границе [5]

$$
E(R) \geqslant \sup _{s \geqslant 1}\left(\sup _{\pi} \tilde{\mu}(\pi, s)-s R\right) \equiv E_{e x}(R),
$$

с потенциалом

$$
\widetilde{\mu}(\pi, s)=-s \log \sum_{i, k} \pi_{i} \pi_{k}\left|\left\langle\psi_{i} \mid \psi_{k}\right\rangle\right|^{2 / s} .
$$

Поведение функций $E_{r}(R), E_{e x}(R)$ напоминает поведение аналогичных функций в классической теории информации, где $E_{r}(R)$ дает лучшую границу для больших, а $E_{e x}(R)$ для малых скоростей передачи $R$. В промежуточном диапазоне скоростей границы $E_{r}(R), E_{e x}(R)$ совпадают и равны линейной функции $\widetilde{C}-R$, где

$$
\widetilde{C}=\sup _{\pi} \widetilde{\mu}(\pi, 1)=\sup _{\pi} \mu(\pi, 1)=-\log \inf _{\pi} \operatorname{Tr} \bar{S}_{\pi}^{2} .
$$

\section{§ 3. Квантовый двоичный канал}

Максимизация гранищ $E_{r}(\pi, R), E_{e x}(\pi, R)$ по $\pi$, являюшаяся непростой задачей и в классическом случае, еше более трудна в квантовом случае. Однако если распределение $\pi^{0}$, максимизируюшее потенщиал $\mu(\pi, s)$ или $\widetilde{\mu}(\pi, s)$, не зависит от $s$, то

$$
E_{r}(R)=E_{r}\left(\pi^{0}, R\right), \quad E_{e x}(R)=E_{e x}\left(\pi^{0}, R\right) .
$$

Такая ситуащия имеет место для двоичного канала с чистыми состояниями.

Пусть $\left|\psi_{0}\right\rangle,\left|\psi_{1}\right\rangle$ - векторы состояний в двумерном унитарном пространстве $\mathscr{H}$. Обозначим $\left|\left\langle\psi_{0} \mid \psi_{1}\right\rangle\right|=\varepsilon$. Собственные числа оператора плотности $\bar{S}_{\pi}=(1-\pi) S_{0}+$ $\pi S_{1}$, где в обозначениях распределение $\pi$ идентифицируется с вероятностью символа 1 , имеют вид

$$
\begin{aligned}
& \lambda_{1}=\frac{1}{2}\left[1-\sqrt{1-4\left(1-\varepsilon^{2}\right) \pi(1-\pi)}\right], \\
& \lambda_{2}=\frac{1}{2}\left[1+\sqrt{1-4\left(1-\varepsilon^{2}\right) \pi(1-\pi)}\right] .
\end{aligned}
$$

Для любого фиксированного $s$ потенциалы

$$
\begin{aligned}
& \mu(\pi, s)=-\log \left(\lambda_{1}^{1+s}+\lambda_{2}^{1+s}\right), \\
& \widetilde{\mu}(\pi, s)=-s \log \left(\pi^{2}+(1-\pi)^{2}+2 \pi(1-\pi) \varepsilon^{2 / s}\right)
\end{aligned}
$$

максимизируются при $\pi=1 / 2$. Обозначая

$$
\begin{aligned}
& \mu(s)=\mu(1 / 2, s)=-\log \left[\left(\frac{1-\varepsilon}{2}\right)^{1+s}+\left(\frac{1+\varepsilon}{2}\right)^{1+s}\right], \\
& \widetilde{\mu}(s)=\widetilde{\mu}(1 / 2, s)=-s \log \left[\frac{1+\varepsilon^{2 / s}}{2}\right]
\end{aligned}
$$


получаем нижнюю границу для квантовой функции надежности в следующем виде:

$$
\begin{aligned}
& E(R) \geqslant \widetilde{\mu}\left(\widetilde{s}_{R}\right)-\widetilde{s}_{R} R, \quad 0<R \leqslant \widetilde{\mu}^{\prime}(1) ; \\
& E(R) \geqslant \widetilde{C}-R, \quad \widetilde{\mu}^{\prime}(1) \leqslant R \leqslant \mu^{\prime}(1) ; \\
& E(R) \geqslant \mu\left(s_{R}\right)-s_{R} R, \quad \mu^{\prime}(1) \leqslant R<C,
\end{aligned}
$$

где $\widetilde{s}_{R}, s_{R}$ - решения уравнений $\widetilde{\mu}^{\prime}\left(\widetilde{s}_{R}\right)=R, \mu^{\prime}\left(s_{R}\right)=R$, а значения констант даются формулами

$$
\begin{aligned}
\widetilde{C} & =\mu(1)=\widetilde{\mu}(1)=-\log \left(\frac{1+\varepsilon^{2}}{2}\right), \\
\widetilde{\mu}^{\prime}(1) & =\widetilde{\mu}(1)+\frac{\varepsilon^{2} \log \varepsilon^{2}}{1+\varepsilon^{2}} \\
\mu^{\prime}(1) & =-\frac{(1-\varepsilon)^{2} \log \left(\frac{1-\varepsilon}{2}\right)+(1+\varepsilon)^{2} \log \left(\frac{1+\varepsilon}{2}\right)}{2\left(1+\varepsilon^{2}\right)} \\
C & =\mu^{\prime}(0)=-\left[\left(\frac{1-\varepsilon}{2}\right) \log \left(\frac{1-\varepsilon}{2}\right)+\left(\frac{1+\varepsilon}{2}\right) \log \left(\frac{1+\varepsilon}{2}\right)\right] .
\end{aligned}
$$

Максимальное количество информации $C_{1}$, которое можно получить за один шаг, достигается для равномерного распределения на входе $(\pi=1 / 2)$ и для решаюшего правила (27), которое в данном случае определяется ортонормированным базисом в $\mathscr{H}$, расположенным симметрично относительно векторов $\left|\psi_{0}\right\rangle,\left|\psi_{1}\right\rangle[36],[30]$, и совпадает с пропускной способностью классического двоичного симметричного канала с вероятностью ошибки $\left(1-\sqrt{1-\varepsilon^{2}}\right) / 2$,

$$
C_{1}=\frac{1}{2}\left[\left(1+\sqrt{1-\varepsilon^{2}}\right) \log \left(1+\sqrt{1-\varepsilon^{2}}\right)+\left(1-\sqrt{1-\varepsilon^{2}}\right) \log \left(1-\sqrt{1-\varepsilon^{2}}\right)\right] .
$$

Сравнение этой величины с $\widetilde{C}$ показьвает, что $C_{1}<\widetilde{C}$ при $0<\varepsilon<1$ (хотя разность между этими функциями весьма мала, см. рис. 1 , где использована двоичная шкала для информационных величин). Поскольку $C \geqslant \widetilde{C}$, отсюда следует строгая супераддитивность $C_{n}>n C_{1}$. Однако нахождение практических блочных кодов, реализуюших в полной мере это свойство, является трудной задачей, см. [41].

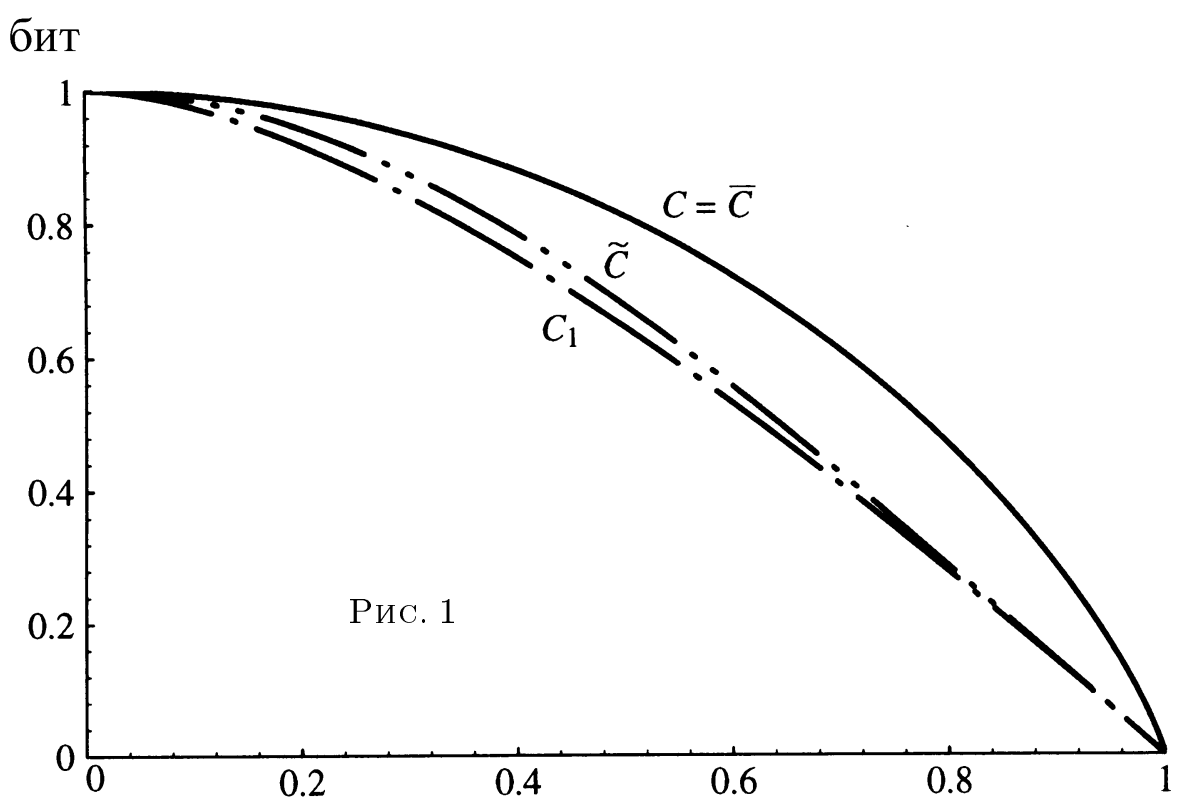


Нетривиальность этой проблемы хорошо иллюстрируется следующим простым примером. Из энтропийной гранищы следует, что значение $C=\log 2$ является абсолютным максимумом пропускной способности для всех каналов, использующих состояния в двумерном унитарном пространстве, причем это значение очевидным образом достигается для бинарного канала с ортогональньми состояниями. Для бинарного канала с неортогональными состояниями $C<\log 2$. Рассмотрим теперь канал с тремя чистьми состояниями, задаваемыми векторами $\left|\psi_{1}\right\rangle,\left|\psi_{2}\right\rangle,\left|\psi_{3}\right\rangle$ такими, что $\left\langle\psi_{i} \mid \psi_{j}\right\rangle=-1 / 2$ при $i \neq j$. Полагая $\pi_{i}=1 / 3$, имеем $\bar{S}_{\pi}=\frac{1}{2} I$, откуда $C=H\left(\bar{S}_{\pi}\right)=\log 2$, несмотря на то, что среди сигнальных состояний нет ни одной пары ортогональных. Можно ли, однако, предложить конструктивньй код, позволяюший сколь-нибудь приблизиться к этому теоретическому пределу?

\section{§4. Случай произвольных состояний с ограниченной энтропией}

Случай произвольных сигнальных состояний значительно более сложен; в частности, до сих пор не удается правильным образом обобшить геометрическую границу (29) для чистых состояний. Вместо этого доказательство прямой теоремы кодирования в случае произвольных сигнальных состояний, данное в [26], а также в [42]), основьвается на некотором обобшении понятия "совместно типичных" последовательностей в классической теории информации, см. [8]. Это реализуется выбором в выражении (25) квантового решаюшего правила

$$
X_{w^{k}}=\left(\sum_{l=1}^{M} P P_{w^{l}} P\right)^{-1 / 2} P P_{w^{k}} P\left(\sum_{l=1}^{M} P P_{w^{l}} P\right)^{-1 / 2}
$$

где $P$ - проектор на типичное подпространство оператора

$$
\bar{S}_{\pi}=\sum_{i \in A} \pi_{i} S_{i}
$$

a $P_{w^{k}}$ - проектор на подходящую модификацию типичного подпространства для оператора плотности $S_{w^{k}}$. Именно, в качестве $P_{w^{k}}$ выбирается спектральньй проектор оператора $S_{w^{k}}$, отвечаюший собственным числам $\lambda_{J}$ в интервале $\left(\mathrm{e}^{-n\left[\bar{H}_{\pi}\left(S_{(\cdot)}\right)+\delta\right]}, \mathrm{e}^{n\left[\bar{H}_{\pi}\left(S_{(\cdot)}\right)-\delta\right]}\right)$. Оператор $P_{w^{k}}$ обладает следуюшими основными свойствами:

$$
\begin{gathered}
P_{w^{k}} \leqslant S_{w^{k}} \mathrm{e}^{n\left[\bar{H}_{\pi}\left(S_{(\cdot)}\right)+\delta\right]}, \\
\quad \mathrm{E} \operatorname{Tr} S_{w^{k}}\left(I-P_{w^{k}}\right) \leqslant \epsilon .
\end{gathered}
$$

Оператор $\left(\sum_{l=1}^{M} P P_{w^{l}} P\right)^{-1 / 2}$ в $(44)$ определяется как обобщенньй обратный оператора $\left(\sum_{l=1}^{M} P P_{w^{l}} P\right)^{1 / 2}$, и равен 0 на нулевом подпространстве этого оператора, содержащем в себе область значений проектора $I-P$. Обозначая $\widehat{P}$ проектор на область значений оператора $\sum_{l=1}^{M} P P_{w^{l}} P$, имеем

$$
P P_{w^{l}} P \leqslant \widehat{P} \leqslant P, \quad l=1, \ldots, M \text {. }
$$


По сравнению с первоначальным доказательством, данным в [26], [42], приводимое ниже доказательство более непосредственно ведет к цели, не требуя использования спектральных представлений операторов $S_{w^{k}}$.

Теорема 3. Пропускная способность с-q канала $i \rightarrow S_{i}$, удовлетворяющего условию (24), дается выражением

$$
C=\bar{C} \equiv \sup _{\pi} \Delta H(\pi)
$$

ДокАЗАТеЛЬСтво. Рассмотрим случай, когда супремум конечен (в противном случае доказательство легко модифицировать). В силу доказанного в $\S$ II.3 достаточно показать, что

$$
p\left(n, \mathrm{e}^{n R}\right) \rightarrow 0 \text { для } R<\bar{C} .
$$

Для упрошения обозначений мы в дальнейшем будем нумеровать слова индексом $w$, опуская $k$.

Обозначая $A_{w}=P_{w} P\left(\sum_{w^{\prime}=1}^{M} P P_{w^{\prime}} P\right)^{-1 / 2}$ и используя некоммутативный аналог неравенства Коши-Буняковского

$$
\left|\operatorname{Tr} S_{w} A_{w}\right|^{2} \leqslant \operatorname{Tr} S_{w} A_{w}{ }^{*} A_{w}
$$

получаем

$$
\bar{\lambda}(W, X) \leqslant \frac{1}{M} \sum_{w=1}^{M}\left[1-\left|\operatorname{Tr} S_{w} A_{w}\right|^{2}\right] \leqslant \frac{2}{M} \sum_{w=1}^{M}\left[1-\operatorname{Tr} S_{w} A_{w}\right],
$$

где $\operatorname{Tr} S_{w} A_{w}=\operatorname{Tr} S_{w} P_{w}\left(\sum_{w^{\prime}=1}^{M} P P_{w^{\prime}} P\right)^{-1 / 2}$ является вешественным числом, заключенным между 0 и 1 . Применяя неравенство

$$
-2 x^{-1 / 2} \leqslant-3+x, \quad x>0,
$$

вытекающее из (37), получаем, учитьвая (47)

$$
-2\left(\sum_{w^{\prime}=1}^{M} P P_{w^{\prime}} P\right)^{-1 / 2} \leqslant-3 P+\sum_{w^{\prime}=1}^{M} P P_{w^{\prime}} P \leqslant-3 P P_{w} P+\sum_{w^{\prime}=1}^{M} P P_{w^{\prime}} P .
$$

Поэтому

$$
\begin{aligned}
\bar{\lambda}(W, X) & \leqslant \frac{1}{M} \sum_{w=1}^{M}\left[2 \operatorname{Tr} S_{w}-3 \operatorname{Tr} S_{w} P_{w} P P_{w} P+\sum_{w^{\prime}=1}^{M} \operatorname{Tr} S_{w} P_{w} P P_{w^{\prime}} P\right] \\
& =\frac{1}{M} \sum_{w=1}^{M}\left[2 \operatorname{Tr} S_{w}\left(I-P_{w} P P_{w} P\right)+\sum_{w^{\prime}: w^{\prime} \neq w} \operatorname{Tr} S_{w} P_{w} P P_{w^{\prime}} P\right] .
\end{aligned}
$$


Принимая во внимание оценку

$$
\begin{aligned}
\operatorname{Tr} S_{w}\left(I-P_{w} P P_{w} P\right)= & \operatorname{Tr} S_{w}\left(I-P_{w}\right) P P_{w} P+\operatorname{Tr} S_{w}(I-P) P_{w} \\
& -\operatorname{Tr} S_{w}(I-P) P_{w}(I-P)+\operatorname{Tr} S_{w}\left(I-P_{w}\right) P+\operatorname{Tr} S_{w}(I-P) \\
\leqslant & 2\left[\operatorname{Tr} S_{w}\left(I-P_{w}\right)+\operatorname{Tr} S_{w}(I-P)\right]
\end{aligned}
$$

получаем конечное неравенство

(50) $\inf _{X} \bar{\lambda}(W, X) \leqslant \frac{1}{M} \sum_{w=1}^{M}\left\{4 \operatorname{Tr} S_{w}(I-P)+4 \operatorname{Tr} S_{w}\left(I-P_{w}\right)+\sum_{w^{\prime}: w^{\prime} \neq w} \operatorname{Tr} P S_{w} P P_{w^{\prime}}\right\}$.

Теперь применим процедуру случайного кодирования, вновь предполагая, что слова $w^{1}, \ldots, w^{M}$ выбираются случайньм образом, независимо друг от друга, с распределением вероятностей (32). Тогда, аналогично соотношению (33), имеем $\mathrm{E} S_{w}=$ $\bar{S}_{\pi}^{\otimes n}$, где $\bar{S}_{\pi}=\sum_{i} \pi_{i} S_{i}$. Усредняя неравенство (50) и учитьвая независимость операторов $S_{w}, P_{w^{\prime}}$, получаем

$$
\mathrm{E} \inf _{X} \bar{\lambda}(W, X) \leqslant 4 \operatorname{Tr} \bar{S}_{\pi}^{\otimes n}(I-P)+4 \mathrm{E} \operatorname{Tr} S_{w}\left(I-P_{w}\right)+(M-1) \operatorname{Tr} \bar{S}_{\pi}^{\otimes n} P \mathrm{E} P_{w^{\prime}}
$$

Используя неравенства $(36),(46)$, выражаюшие типичность проекторов $P, P_{w}$, и свойства следа операторов, получаем

$$
\operatorname{Einf}_{X} \bar{\lambda}(W, X) \leqslant 8 \epsilon+(M-1)\left\|\bar{S}_{\pi}^{\otimes n} P\right\| \operatorname{Tr} \mathrm{E} P_{w^{\prime}},
$$

для $n \geqslant n(\pi, \epsilon, \delta)$. В силу свойства $(35)$ проектора $P$, имеем

$$
\left\|\bar{S}^{\otimes n} P\right\| \leqslant \mathrm{e}^{-n\left[H\left(\bar{S}_{\pi}\right)-\delta\right]}
$$

и согласно свойству (45) проектора $P_{w}$,

$$
\operatorname{Tr} \mathrm{E} P_{w^{\prime}}=\mathrm{E} \operatorname{Tr} P_{w^{\prime}} \leqslant \mathrm{E} \operatorname{Tr} S_{w^{\prime}} \cdot \mathrm{e}^{n\left[\bar{H}\left(S_{(\cdot)}\right)+\delta\right]}=\mathrm{e}^{n\left[\bar{H}\left(S_{(\cdot)}\right)+\delta\right]} .
$$

Таким образом,

$$
\operatorname{Enf}_{X} \bar{\lambda}(W, X) \leqslant 8 \epsilon+(M-1) \mathrm{e}^{-n\left[H\left(\bar{S}_{\pi}\right)-\bar{H}\left(S_{(\cdot)}\right)-2 \delta\right]}
$$

Выберем распределение $\pi=\pi^{0}$ так, чтобы $\Delta H\left(\pi^{0}\right) \geqslant \bar{C}-\delta$. Тогда

$$
p(n, M) \leqslant 8 \epsilon+(M-1) \mathrm{e}^{-n[\bar{C}-3 \delta]}
$$

при $n \geqslant n\left(\pi^{0}, \epsilon, \delta\right)$. Таким образом, $p\left(n, \mathrm{e}^{n[\bar{C}-4 \delta]}\right) \rightarrow 0$ при $n \rightarrow \infty$, откуда вытекает (49). 


\section{IV. c-q каналы с ограничениями на входе}

\section{§ 1. Теорема кодирования}

Важность рассмотрения каналов с ограничениями на входе была очевидна уже на заре квантовой теории информации: это позволяет, в частности, дать математически корректную формулировку и решение вопросов типа: "Какое максимальное количество информации может быть передано по физическому каналу связи при ограниченных энергетических ресурсах?" (см. $§ \S 2-3)$.

В настоящем обзоре мы ограничимся случаем с-q каналов с классическим ограничением на входе. Рассмотрим сначала канал с дискретным входньм алфавитом $A=$ $\{i\}$. Пусть $f(i)$ - неотрицательная функция на входном алфавите. Введем класс $\mathscr{P}_{1}$ распределений $\pi$ на $A$, удовлетворяющих условию

$$
\sum_{i} f(i) \pi(i) \leqslant E
$$

где $E$ - положительное число. Введем аддитивное ограничение на входные слова $w=$ $\left(i_{1}, \ldots, i_{n}\right)$, полагая

$$
f\left(i_{1}\right)+\cdots+f\left(i_{n}\right) \leqslant n E
$$

и обозначим $\mathscr{P}_{n}$ класс распределений на $A$, удовлетворяюших соответствующему условию

$$
\sum_{i_{1}, \ldots, i_{n}}\left[f\left(i_{1}\right)+\cdots+f\left(i_{n}\right)\right] \pi\left(i_{1}, \ldots, i_{n}\right) \leqslant n E
$$

Определим теперь величины $C, \bar{C}$ аналогично $\S$ II.2, но беря супремум только по распределениям $\pi$ из класса $\mathscr{P}_{n}$, т.е.

$$
C=\lim _{n \rightarrow \infty} \frac{C_{n}}{n}, \quad \bar{C}=\lim _{n \rightarrow \infty} \frac{\bar{C}_{n}}{n},
$$

где

$$
C_{n}=\sup _{\pi \in \mathscr{P}_{n}} \sup _{X} I_{n}(\pi, X), \quad \bar{C}_{n}=\sup _{\pi \in \mathscr{P}_{n}} \Delta H_{n}(\pi),
$$

а $I_{n}(\pi, X), \Delta H_{n}(\pi)$ являются аналогами количества информации (9) и энтропийной гранищы (8) для тензорной степени рассматриваемого с-qканала в пространстве $\mathscr{H}{ }^{\otimes n}$.

Отметим, что, как и в случае канала без ограничений, последовательность $\bar{C}_{n}$ аддитивна, и поэтому

$$
\bar{C}=\sup _{\pi \in \mathscr{P}_{1}} \Delta H(\pi)
$$

В самом деле, достаточно проверить, что

$$
\bar{C}_{n} \leqslant n \bar{C}_{1} .
$$


Субаддитивность квантовой энтропии по отношению к тензорному произведению влечет

$$
\Delta H_{n}(\pi) \leqslant \sum_{k=1}^{n} \Delta H\left(\pi^{(k)}\right)
$$

где $\pi^{(k)}$ есть $k$-е маргинальное распределение $\pi$ на $A$. Кроме того,

$$
\sum_{k=1}^{n} \Delta H\left(\pi^{(k)}\right) \leqslant n \Delta H(\bar{\pi})
$$

где $\bar{\pi}=\frac{1}{n} \sum_{k=1}^{n} \pi^{(k)}$, поскольку $\Delta H(\pi)$ вьпуклая вверх функция $\pi[19]$. Неравенство (54) может быть переписано в виде

$$
\frac{1}{n} \sum_{k=1}^{n} \sum_{i_{k}} f\left(i_{k}\right) \pi^{(k)}\left(i_{k}\right) \leqslant E
$$

откуда следует, что $\bar{\pi} \in \mathscr{P}_{1}$, если $\pi \in \mathscr{P}_{n}$. Беря супремум по $\pi \in \mathscr{P}_{n}$, получаем $(56)$.

Теорема 4. Пропускная способность с-q канала $i \rightarrow S_{i}$ с ограничением на входе (53), удовлетворяющего условию (24), дается выражсением (55).

ДокАЗАтЕльство. Достаточно показать, что если $C<\infty$ и $R>C$, то $p\left(n, \mathrm{e}^{n R}\right)$ $\nrightarrow 0$, а если вьполняется условие $(24)$ и $R<\bar{C}$, то $p\left(n, \mathrm{e}^{n R}\right) \rightarrow 0$.

Доказательство первого утверждения (т.е. обратной теоремы кодирования) основьвается на модификации неравенства (23)

$$
\log M \cdot(1-p(n, M)) \leqslant \sup _{\pi \in \mathscr{P}_{n}} \sup _{X} I_{n}(\pi, X)+1 .
$$

В самом деле, пусть, как в доказательстве теоремы $1, \mathscr{J}$ обозначает случайную величину, описывающую выходные значения канала при использовании решаюшего правила $X$, если слова кода $(W, X)$ выбираются в соответствии с распределением $\pi_{M}$, приписывающим одинаковую вероятность $1 / M$ каждому слову. Рассмотрим неравенство Фано. Поскольку слова кода удовлетворяют условию (53), имеем $\pi_{M} \in \mathscr{P}_{n}$, откуда вытекает неравенство (57). Подставляя $M=\mathrm{e}^{n R}$, получаем первое утверждение теоремы.

В классической теории информации прямая теорема кодирования доказывается применением случайного кодирования с модифицированным распределением вероятностей (32), содержашим дополнительный множитель, выделяющий только слова, для которых ограничение почти превращается в равенство [12, гл. 7.3]. Аналогичный прием может быть применен и в квантовом случае. Пусть $\pi$ - распределение, удовлетворяющее условию (52), а $\mathrm{P}$ - вероятностная мера на множестве наборов $M$ слов, относительно которой слова независимы и имеют распределение (32). Обозначим $\nu_{n}=\mathrm{P}\left(\frac{1}{n} \sum_{k=1}^{n} f\left(i_{k}\right) \leqslant E\right)$ и зададим модифицированную меру таким образом, что слова по-прежнему независимы, но имеют распределение вероятностей

$$
\widetilde{\mathrm{P}}\left(w=\left(i_{1}, \ldots, i_{n}\right)\right)= \begin{cases}\nu_{n}^{-1} \pi_{i_{1}} \cdot \ldots \cdot \pi_{i_{n}}, & \text { если } \sum_{k=1}^{n} f\left(i_{k}\right) \leqslant n E \\ 0 & \text { в противном случае. }\end{cases}
$$


Отметим, что поскольку $\pi \in \mathscr{P}_{1}$, то $\mathrm{E} f \leqslant E$ (где $\mathrm{E}(\widetilde{\mathrm{E}})$ - математическое ожидание, отвечающее вероятностной мере $\mathrm{P}(\widetilde{\mathrm{P}})$ ). Согласно центральной предельной теореме

$$
\lim _{n \rightarrow \infty} \nu_{n} \geqslant \frac{1}{2},
$$

поэтому $\widetilde{E} \xi \leqslant 2^{m} \mathrm{E} \xi$ для любой неотрицательной случайной величины $\xi$, зависяшей от $m$ слов. Для вероятности ошибки (25) была получена верхняя граница (50). Усредним это неравенство относительно Р. Каждое слагаемое в правой части (50) зависит не более чем от двух различных слов, поэтому

$$
\widetilde{\mathrm{E}} \inf _{X} \bar{\lambda}(W, X) \leqslant 4 \mathrm{E} \inf _{X} \bar{\lambda}(W, X),
$$

причем математическое ожидание относительно Р стремится к нулю, когда $M=\mathrm{e}^{n R}$, $n \rightarrow \infty$, с $R<C-3 \delta$. Следовательно, и $\widetilde{\mathrm{E}} \bar{\lambda}$ стремится к нулю при том же условии. Поскольку мера $\widetilde{P}$ сконцентрированана словах, удовлетворяющих условию (53), найдется последовательность кодов размеров $M=\mathrm{e}^{n R}$, для которых вероятность ошибки $\bar{\lambda}(W, X)$ стремится к нулю при $n \rightarrow \infty$.

Этот результат обобщается и на случай непрерывного входного алфавита $A$. Пусть $A$ - сепарабельное локально-компактное топологическое пространство. Предположим, что задано слабо непрерывное отображение $x \rightarrow S_{x}$ алфавита $A$ в множество операторов плотности $\mathscr{S}(\mathscr{H})$ (слабая непрерывность означает непрерьвность матричных элементов $\left\langle\psi\left|S_{x}\right| \phi\right\rangle ; \psi, \phi \in \mathscr{H}$ ). Для наших целей достаточно ограничиться описанием соответствующего c-q канала в терминах этого отображения, хотя не представляет большого труда построить и непрерывный аналог представления (17). Преимущество такого упрощенного описания состоит также в том, что определение кода формулируется при этом совершенно аналогично случаю дискретного алфавита.

Для произвольного борелевского распределения вероятностей $\pi$ на $A$ рассмотрим величину

$$
\Delta H(\pi)=\int_{A} H\left(S_{x} ; \bar{S}_{\pi}\right) \pi(d x),
$$

где

$$
\bar{S}_{\pi}=\int_{A} S_{x} \pi(d x) .
$$

Последний интеграл определен в силу слабой непрерывности функции $S_{x}$ и задает оператор плотности в $\mathscr{H}$. Более того, неотрищательная функция $H\left(S_{x} ; \bar{S}_{\pi}\right)$ полунепрерьвна снизу [47], поэтому интеграл (59) также определен.

Введем аналог условия (24):

$$
\sup _{x \in A} H\left(S_{x}\right)<\infty .
$$

При этом условии имеет место представление (8), где

$$
\bar{H}\left(S_{(\cdot)}\right)=\int_{A} H\left(S_{x}\right) \pi(d x) .
$$


Предположим также, что на $A$ задана неотрицательная борелевская функция $f$, и рассмотрим множество $\mathscr{P}_{1}$ распределений вероятностей $\pi$ на $A$, удовлетворяюших условию

$$
\int_{A} f(x) \pi(d x) \leqslant E
$$

Введем аддитивное ограничение на передаваемые слова, полагая

$$
f\left(x_{1}\right)+\cdots+f\left(x_{n}\right) \leqslant E .
$$

ПреДЛОЖЕНИЕ 2. В предположсении, что отображсение $x \rightarrow S_{x}$ слабо непрерывно, функция $f$ непрерывна снизу и выполняется условие (61), пропускная способность канала с ограничением на входе (63) дается вырахсением

$$
\bar{C}=\sup _{\pi \in \mathscr{P}_{1}} \Delta H(\pi)
$$

Доказательство этого предложения, основанное на теореме 4 и свойствах непрерьвности квантовой энтропии, можно найти в предварительной версии настоящего обзора [28].

\section{§ 2. Гауссовский канал с одной степенью свободы}

Квантовые гауссовские состояния определены для систем, описываемых каноническими коммутационными соотношениями (простое введение можно найти в [25, гл. V]). В настоящем обзоре мы ограничимся калибровочно инвариантными состояниями, обладающими дополнительной комплексной структурой. В этом случае каноническое коммутационное соотношение для системы с одной степенњю свободы может быть записано в виде

$$
\left[a, a^{\dagger}\right] \subseteq I
$$

где $a, a^{\dagger}$ - операторы уничтожения и рождения, а $\mathscr{H}$ есть пространство неприводимого представления этого соотношения.

Пусть алфавит $A$ есть комплексная плоскость $\mathbb{C}$, и пусть для каждого $\alpha \in \mathbb{C}$ оператор плотности $S_{\alpha}$ задается соотношением

$$
S_{\alpha}=\frac{1}{\pi N} \int \exp \left(-\frac{|z-\alpha|^{2}}{N}\right)|z\rangle\langle z| d^{2} z,
$$

где $N$ - положительное число (среднее число квантов), а $|z\rangle$ векторы когерентных состояний, т.е. собственные векторы оператора уничтожения, $a|z\rangle=z|z\rangle$, образуюшие переполненную систему в $\mathscr{H}$. Первые и вторые моменты состояния $S_{\alpha}$ даются выражениями

$$
\operatorname{Tr} S_{\alpha} a=\alpha, \quad \operatorname{Tr} S_{\alpha} a^{\dagger} a=N+|\alpha|^{2} .
$$

Напомним также, что

$$
S_{\alpha}=V(\alpha) S_{0} V(\alpha)^{*},
$$


где

$$
V(\alpha)=\exp \left(\alpha a^{\dagger}-\bar{\alpha} a\right)
$$

- унитарные операторы Вейля, и что спектральное разложение $S_{0}$ имеет вид

$$
S_{0}=\frac{1}{N+1} \sum_{n=0}^{\infty}\left(\frac{N}{N+1}\right)^{n}|n\rangle\langle n|,
$$

где $|n\rangle$ - собственные векторы оператора числа квантов $a^{\dagger} a$, отвечаюшие собственньм значениям $n=0,1, \ldots$ В частности, состояния (65) имеют одинаковую энтропию

$$
H\left(S_{\alpha}\right)=(N+1) \log (N+1)-N \log N \equiv g(N),
$$

где $g(x)$ - непрерьвная вогнутая монотонно возрастающая функция $x \geqslant 0$. Известно, что состояния $S_{\alpha}$ обладают максимальной энтропией среди всех состояний, удовлетворяющих соотношениям (66). Это вытекает из следующей леммы.

Лемма 1. Оператор (67) имеет максимальную энтропию среди всех операторов плотности $S$, удовлетворяющих условию

$$
\operatorname{Tr} S a^{\dagger} a \leqslant N
$$

ДокАЗАТЕЛЬСТво. Обозначим $S^{\prime}$ оператор плотности, диагональный в базисе $\{|n\rangle\}$, с элементами $s_{n}=\langle n|S| n\rangle$ на диагонали. Этот оператор удовлетворяет условию (69), и

$$
H\left(S^{\prime}\right)-H(S)=H\left(S ; S^{\prime}\right) \geqslant 0 .
$$

Поэтому максимум достигается на диагональных операторах. Таким образом, надо максимизировать $H(S)=-\sum_{n} s_{n} \log s_{n}$ при условиях $s_{n} \geqslant 0, \sum_{n} s_{n}=1$, и условии $(69)$, которое принимает вид $\sum_{n} n s_{n} \leqslant N$. Применяя метод Лагранжа, получаем решение (67).

Поскольку оператор (67) удовлетворяет условиям (66) (при $\alpha=0$ ), он обладает также максимальной энтропией среди операторов, удовлетворяюших этому условию.

Рассмотрим теперь с-q канал $\alpha \rightarrow S_{\alpha}$, которьй является квантовьм аналогом канала с аддитивньм гауссовским шумом (см. [14], [16], [22]). Условие (61) выполнено здесь тривиальным образом, и для любого распределения $\pi\left(d^{2} \alpha\right)$ на входе канала

$$
\Delta H(\pi)=H\left(\bar{S}_{\pi}\right)-H\left(S_{0}\right)
$$

где

$$
\bar{S}_{\pi}=\int S_{\alpha} \pi\left(d^{2} \alpha\right)
$$

Введем аддитивное ограничение (63) на входе, полагая $f(\alpha)=|\alpha|^{2}$. Таким образом, множество $\mathscr{P}_{1}$ определено соотношением

$$
\int|\alpha|^{2} \pi\left(d^{2} \alpha\right) \leqslant E
$$


Физический смысл константы $E$ - "среднее число квантов" сигнала, в рассматриваемом случае одной степени свободы пропорциональное энергии сигнала. В силу (66), ограничение (70) влечет

$$
\operatorname{Tr} \bar{S}_{\pi} a^{\dagger} a \leqslant N+E .
$$

Согласно лемме 1 , максимум энтропии

$$
H\left(\bar{S}_{\pi}\right)=g(N+E)
$$

достигается на гауссовском операторе плотности

$$
\bar{S}_{\pi}=\frac{1}{\pi(N+E)} \int \exp \left(-\frac{|z|^{2}}{(N+E)}\right)|z\rangle\langle z| d^{2} z,
$$

отвечающем оптимальному распределению вероятностей

$$
\pi\left(d^{2} \alpha\right)=\frac{1}{\pi E} \exp \left(-\frac{|\alpha|^{2}}{E}\right) d^{2} \alpha .
$$

Согласно предложению 2, пропускная способность канала равна

$$
\begin{aligned}
C=\bar{C} & \equiv g(N+E)-g(N) \\
& =\log \left(1+\frac{E}{N+1}\right)+(N+E) \log \left(1+\frac{1}{N+E}\right)-N \log \left(1+\frac{1}{N}\right) .
\end{aligned}
$$

Это выражение было предложено еще в работе [14] (соотношение (4.28)) в качестве гипотетической верхней границы количества информации, передаваемого квантовым гауссовским каналом. С другой стороны, было показано [13], [34], что эта величина равна пропускной способности квазиклассического “фотонного канала связи”. Приведенное выше рассуждение, основьваюшееся на теореме 4 и предложении 2 , впервые дает доказательство асимптотической эквивалентности, в смысле пропускной способности, гауссовского с-q канала и этого квазиклассического канала. Для большей ясности приведем описание фотонного канала.

Рассмотрим дискретное семейство состояний

$$
S_{m}=P(m) S_{0} P(m)^{*}, \quad m=0,1, \ldots,
$$

где $P(m)$ - оператор энергетического сдвига, определяемьй соотношением $P(m)|n\rangle=$ $|n+m\rangle$. Отметим, что $P(m)=P^{m}$, где $P$ - изометрический оператор, сопряженный к оператору фазы [25]. Состояния $S_{m}$ имеют одинаковую энтропию (68), совпадаюшую с энтропией состояний $S_{\alpha}$, и среднее число квантов, равное

$$
\operatorname{tr} S_{m} a^{\dagger} a=N+m .
$$

Все операторы (75) диагональны в базисе $\{|n\rangle\}$, так что канал $m \rightarrow S_{m}$ является квазиклассическим. 
Налагая ограничение

$$
\sum_{m=0}^{\infty} m \pi_{m} \leqslant E
$$

где $\pi_{m}$ - распределение на входе, и вводя оператор плотности

$$
\bar{S}_{\pi}^{\prime}=\sum_{m=0}^{\infty} \pi_{m} S_{m}
$$

в силу (76) получаем для него то же условие (71). Максимум энтропии, равньй (72), вновь достигается на операторе $(73)$, спектральное разложение которого имеет вид

$$
\bar{S}_{\pi}=\frac{1}{N+E+1} \sum_{n=0}^{\infty}\left(\frac{N+E}{N+E+1}\right)^{n}|n\rangle\langle n| .
$$

Максимум достигается на распределении [34]

$$
\pi_{m}=\frac{N}{N+E} \delta_{m 0}+\frac{E}{N+E}\left[\frac{1}{N+E+1}\left(\frac{N+E}{N+E+1}\right)^{m}\right], \quad m=0,1, \ldots
$$

Рассмотрим случай чистых состояний, когда $S_{0}=|0\rangle\langle 0|$. Тогда фотонный канал вообще является детерминированньм и допускает безошибочную передачу; его функция надежности равна $+\infty$. Соответствуюший гауссовский канал использует неортогональные когерентные состояния и нетривиальную функцию надежности, см. [27], и тем не менее позволяет асимптотически безошибочную передачу с той же скоростью, что и фотонный канал.

\section{§ 3. Классический сигнал на фоне квантового шума}

Перейдем к рассмотрению более реалистичной динамической модели квантового гауссовского канала. Подобно классическому случаю, мы используем прием сведения случайного процесса к набору параллельных каналов с одной степенью свободы $([12, \S 8.3])$, однако здесь такое сведение несет дополнительную нагрузку, как инструмент квантования случайного процесса. Возможна формулировка задачи непосредственно в терминах квантового случайного процесса, но вычисление пропускной способности, основьвающееся на такой формулировке, является пока открытым вопросом (см. обсуждение в конце этого раздела).

Рассмотрим периодическую операторнозначную функцию

$$
X(t)=\sum_{j} \sqrt{\frac{2 \pi \hbar \omega_{j}}{T}}\left(a_{j} \mathrm{e}^{-i \omega_{j} t}+a_{j}^{\dagger} \mathrm{e}^{i \omega_{j} t}\right), \quad t \in[0, T]
$$

где $[0, T]$ интервал наблюдения,

$$
\omega_{j}=\frac{2 \pi j}{T}, \quad j=1,2, \ldots
$$


- набор частот (зависяший от $T$ ) $, a_{j}^{\dagger}, a_{j}$ - операторы рождения-уничтожения, описываюшие $j$-ю степень свободы. В квантовой электродинамике аналогичное соотношение используется для представления компоненты электрического поля в плоской волне с периодическими граничньми условиями. Чтобы избежать проблем, связанных с рассмотрением бесконечного числа степеней свободы, мы ограничим диапазон суммирования в (79) конечным множеством $I_{T}$. В случае ограниченного диапазона частот $0<\underline{\omega}<\omega<\bar{\omega}<\infty$ примем

$$
I_{T}=\left\{j: \underline{\omega} \leqslant \omega_{j} \leqslant \bar{\omega}\right\} .
$$

В случае $\underline{\omega}=0, \bar{\omega}=\infty$ положим

$$
I_{T}=\left\{j: \underline{\omega}_{T} \leqslant \omega_{j} \leqslant \bar{\omega}_{T}\right\}
$$

где $\underline{\omega}_{T} \downarrow 0, \bar{\omega}_{T} \uparrow \infty$ при $T \rightarrow \infty$.

Отметим соотношение

$$
\frac{1}{4 \pi} \int_{0}^{T} X(t)^{2} d t=\sum_{j} \hbar \omega_{j}\left(a_{j}^{\dagger} a_{j}+\frac{1}{2}\right)
$$

для соответствуюшего оператора энергии.

Будем предполагать, что $j$-я степень свободы описывается гауссовским состоянием $(65)$, которое теперь будем обозначать $S_{j}\left(\alpha_{j}\right)$, с первыми и вторыми моментами

$$
\operatorname{Tr} S_{j}\left(\alpha_{j}\right) a_{j}=\alpha_{j}, \quad \operatorname{Tr} S_{j}\left(\alpha_{j}\right) a_{j}^{\dagger} a_{j}=N_{j}+\left|\alpha_{j}\right|^{2} .
$$

Вводя вектор $\alpha=\left(\alpha_{j}\right)$, рассмотрим состояние

$$
S_{\alpha}=\bigotimes_{j} S_{j}\left(\alpha_{j}\right)
$$

описьвающее процесс $X(t)$, так что

$$
\begin{gathered}
\operatorname{Tr} S_{\alpha} X(t)=\alpha(t) \\
\operatorname{Tr} S_{\alpha} \frac{1}{4 \pi} \int_{0}^{T} X(t)^{2} d t=\sum_{j} \hbar \omega_{j}\left(N_{j}+\frac{1}{2}\right)+\frac{1}{4 \pi} \int_{0}^{T} \alpha(t)^{2} d t
\end{gathered}
$$

где

$$
\begin{gathered}
\alpha(t)=\sum_{j} \sqrt{\frac{2 \pi \hbar \omega_{j}}{T}}\left(\alpha_{j} \mathrm{e}^{-i \omega_{j} t}+\bar{\alpha}_{j} \mathrm{e}^{i \omega_{j} t}\right), \\
\frac{1}{4 \pi} \int_{0}^{T} \alpha(t)^{2} d t=\sum_{j} \hbar \omega_{j}\left|\alpha_{j}\right|^{2} .
\end{gathered}
$$


Таким образом, сигнал представляется вешественной функцией $\alpha(t)$. Введем ограничение на среднюю мощность сигнала, полагая

$$
\frac{1}{4 \pi} \int_{0}^{T} \alpha(t)^{2} d t \leqslant T E .
$$

Кодом $(W, X)$ будем назьвать набор $\left(\alpha^{1}(\cdot), X_{1}\right), \ldots,\left(\alpha^{M}(\cdot), X_{M}\right)$, где $\alpha^{k}(\cdot)-$ функции аргумента $t \in[0, T]$, представляющие различные значения сигнала; пропускная способность определяется как супремум значений скорости передачи $R$, для которых инфимум средней вероятности ошибки

$$
\bar{\lambda}(W, X)=\frac{1}{M} \sum_{j=1}^{M}\left(1-\operatorname{Tr} S_{\alpha^{j}} X_{j}\right)
$$

по всем кодам размера $M=\mathrm{e}^{T R}$ стремится к нулю при $T \rightarrow \infty$.

В дальнейшем используется обозначение $(y)_{+}=\max (y, 0)$.

Теорема 5. Предположим, что $N_{j}=N\left(\omega_{j}\right)$, где $N(\omega)$ - непрерывная функиия. Определенная выше пропускная способность равна

$$
C=\frac{1}{2 \pi} \int_{\underline{\omega}}^{\bar{\omega}}\left(g\left(N_{\theta}(\omega)\right)-g(N(\omega))\right)_{+} d \omega,
$$

$2 \partial e$

$$
N_{\theta}(\omega)=\frac{1}{\mathrm{e}^{\theta \hbar \omega}-1}, \quad \omega>0
$$

- распределение Планка, а $\theta$ находится из уравнения

$$
\frac{1}{2 \pi} \int_{\underline{\omega}}^{\bar{\omega}} \hbar \omega\left(N_{\theta}(\omega)-N(\omega)\right)_{+} d \omega=E .
$$

ДоКАЗАТЕЛЬСТВо. Начнем с рассмотрения случая ограниченного диапазона частот. Докажем сначала обратную теорему кодирования: $\inf _{W, X} \bar{\lambda}(W, X) \nrightarrow 0$ при $R>C$. Из неравенства (57) и энтропийной границы вытекает, что

$$
T R \cdot\left(1-\inf _{W, X} \bar{\lambda}(W, X)\right) \leqslant C_{T}+1
$$

где

$$
C_{T}=\sup _{\pi \in \mathscr{P}_{1}} \Delta H(\pi)=\sup _{\pi \in \mathscr{P}_{1}}\left[H\left(\bar{S}_{\pi}\right)-H\left(S_{0}\right)\right]
$$

а множество распределений вероятностей $\mathscr{P}_{1}$ определено соотношением

$$
\int\left(\sum_{j} \hbar \omega_{j}\left|\alpha_{j}\right|^{2}\right) \pi\left(d^{2} \alpha\right) \leqslant E T .
$$


Используя лемму 1 , нетрудно показать, что достаточно ограничиться гауссовскими распределениями $\pi$. Обозначая

$$
\int \hbar \omega_{j}\left|\alpha_{j}\right|^{2} \pi\left(d^{2} \alpha_{j}\right)=m_{j}
$$

имеем

$$
C_{T}=\max \sum_{j \in I_{T}}\left[g\left(N_{j}+m_{j}\right)-g\left(N_{j}\right)\right]
$$

причем максимум берется по множеству

$$
m_{j} \geqslant 0, \quad \frac{1}{2 \pi} \sum_{j \in I_{T}} \hbar \omega_{j} m_{j} \Delta \omega_{j} \leqslant E
$$

где введено обозначение

$$
\Delta \omega_{j}=\frac{2 \pi}{T}
$$

Вводя кусочно-постоянную функцию

$$
N_{T}(\omega)=N_{j}, \quad \omega_{j-1}<\omega<\omega_{j},
$$

имеем

$$
\frac{C_{T}}{T} \leqslant \frac{1}{2 \pi} \max _{\mathscr{M}(\underline{\omega}, \bar{\omega})} \int_{\underline{\omega}}^{\bar{\omega}}\left[g\left(N_{T}(\omega)+m(\omega)\right)-g\left(N_{T}(\omega)\right)\right] d \omega,
$$

где

$$
\mathscr{M}(\underline{\omega}, \bar{\omega})=\left\{m(\cdot): m(\omega) \geqslant 0 ; \quad \frac{1}{2 \pi} \int_{\underline{\omega}}^{\bar{\omega}} \hbar \omega m(\omega) d \omega \leqslant E\right\} .
$$

Поскольку функция $N(\omega)$ равномерно непрерывна на $[\underline{\omega}, \bar{\omega}]$, семейство $N_{T}(\omega)$ равномерно сходится к $N(\omega)$ при $T \rightarrow \infty$. Отсюда следует, что

$$
\limsup _{T \rightarrow \infty} \frac{C_{T}}{T} \leqslant \max _{\mathscr{M}(\underline{\omega}, \omega)} \frac{1}{2 \pi} \int_{\underline{\omega}}^{\bar{\omega}}[g(N(\omega)+m(\omega))-g(N(\omega))] d \omega .
$$

Используя условия Куна-Таккера, нетрудно показать, что максимум достигается при

$$
m^{*}(\omega)=\left(N_{\theta}(\omega)-N(\omega)\right)_{+}
$$

и равен величине $C$, определенной соотношениями (89)-(91). Поэтому из (92) мы заключаем, что $\inf _{W, X} \bar{\lambda}(W, X) \nrightarrow 0$ для $R>C$.

Покажем теперь, что средняя вероятность ошибки стремится к нулю, если $R<C$. Рассмотрим гауссовское распределение

$$
\pi\left(d^{2} \alpha\right)=\exp \left(-\sum_{j} \frac{\left|\alpha_{j}\right|^{2}}{m_{j}^{*}}\right) \prod_{j} d^{2} \alpha_{j}
$$


где

$$
m_{j}^{*}=\left(N_{\theta}\left(\omega_{j}\right)-N_{j}\right)_{+}
$$

и $\theta$ выбрано так, что

$$
\frac{1}{2 \pi} \sum_{j \in I_{T}} \hbar \omega_{j} m_{j}^{*} \Delta \omega_{j}=E
$$

$\left(\right.$ Если $m_{j}^{*}=0$, то в (97) имеется в виду распределение, вырожденное в точке 0.) Применяя основную оценку (50) с длиной слова $n=1$ и с заменой $\delta$ на $\delta T$, получаем

(100) $\inf _{X} \bar{\lambda}(W, X) \leqslant \frac{1}{M} \sum_{j=1}^{M}\left\{4 \operatorname{Tr} S_{\alpha^{j}}(I-P)+4 \operatorname{Tr} S_{\alpha^{j}}\left(I-P_{\alpha^{j}}\right)+\sum_{k \neq j} \operatorname{Tr} P S_{\alpha^{j}} P P_{\alpha^{k}}\right\}$,

где $P$ - спектральный проектор оператора $\bar{S}_{\pi}$, отвечающий интервалу

$$
\left(\mathrm{e}^{-\left[H\left(\bar{S}_{\pi}\right)+\delta T\right]}, \mathrm{e}^{-\left[H\left(\bar{S}_{\pi}\right)-\delta T\right]}\right),
$$

а $P_{\alpha}$ - спектральньй проектор оператора $S_{\alpha}$, отвечающий интервалу

$$
\left(\mathrm{e}^{-\left[\bar{H}\left(S_{(\cdot)}\right)+\delta T\right]}, \mathrm{e}^{-\left[\bar{H}\left(S_{(\cdot)}\right)-\delta T\right]}\right) .
$$

Поскольку операторы плотности $S_{\alpha}$ унитарно эквивалентны $S_{0}$, то $\bar{H}\left(S_{(\cdot)}\right)=H\left(S_{0}\right)$ и среднее слагаемое в неравенстве (100) равно

$$
\operatorname{Tr} S_{0}\left(I-P_{0}\right) .
$$

Имеем

$$
\operatorname{Tr} S_{0}\left(I-P_{0}\right)=\operatorname{Pr}\left\{\left|-\log \lambda_{(\cdot)}-H\left(S_{0}\right)\right| \geqslant \delta T\right\}
$$

где $\operatorname{Pr}$ обозначает распределение, задаваемое собственными числами $\lambda_{(\cdot)}$ оператора плотности $S_{0}$. Согласно неравенству Чебышёва, эта вероятность не превосходит $\mathrm{D}\left(\log \lambda_{(\cdot)}\right) / \delta^{2} T^{2}$. С другой стороны,

$$
\mathrm{D}\left(\log \lambda_{(\cdot)}\right)=\sum_{j} \mathrm{D}_{j}\left(\log \lambda_{(\cdot)}\right),
$$

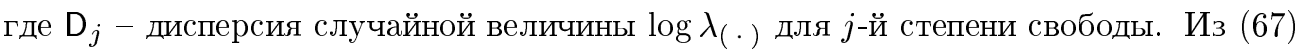
видно, что собственные числа оператора $S_{j}(0)$ суть

$$
\lambda_{n}^{j}=\frac{N_{j}^{n}}{\left(N_{j}+1\right)^{n+1}} ; \quad n=0,1, \ldots,
$$


поэтому

$$
\begin{aligned}
\mathrm{D}_{j}\left(\log \lambda_{(\cdot)}\right) & =\sum_{n=0}^{\infty}\left(-\log \lambda_{n}^{j}-H\left(S_{0}\right)\right)^{2} \lambda_{n}^{j} \\
& =\log ^{2} \frac{N_{j}+1}{N_{j}} \sum_{n=0}^{\infty}\left(n-N_{j}\right)^{2} \frac{N_{j}^{n}}{\left(N_{j}+1\right)^{n+1}}=F\left(N_{j}\right),
\end{aligned}
$$

где

$$
F(x)=x(x+1) \log ^{2} \frac{x+1}{x}
$$

ограниченная функция на $(0, \infty)$. Окончательно,

$$
\operatorname{Tr} S_{0}\left(I-P_{0}\right) \leqslant \frac{\sum_{j} F\left(N_{j}\right)}{\delta^{2} T^{2}}
$$

Заметим, что аналогичная оценка имеет место для $\operatorname{Tr} \bar{S}_{\pi}(I-P)$ с заменой $N_{j}$ на $N_{j}+m_{j}^{*}$.

Пусть теперь слова $\alpha^{1}, \ldots, \alpha^{M}$ выбираются случайным образом, с совместньм распределением $\widetilde{\mathrm{P}}$, которое определяется аналогично соотношению (58), исходя из распределения $\mathrm{P}$, относительно которого слова независимы и имеют одинаковое распределение $\pi\left(d^{2} \alpha\right)$. Тогда $\widetilde{\mathrm{E}} \xi \leqslant 2^{m} \mathrm{E} \xi$ для любой неотрицательной случайной величины $\xi$, зависящей от $m$ слов. Поэтому из (100) получаем

$$
\begin{aligned}
\underset{X}{\widetilde{\mathrm{E}}} \inf _{X} & \bar{\lambda}(W, X) \\
& \leqslant \frac{1}{M} \sum_{j=1}^{M}\left\{8 \mathrm{E} \operatorname{Tr} S_{\alpha^{(j)}}(I-P)+4 \operatorname{Tr} S_{0}\left(I-P_{0}\right)+\sum_{k \neq j} 4 \mathrm{E} \operatorname{Tr} P S_{\alpha^{(j)}} P P_{\alpha^{(k)}}\right\} \\
& =8 \operatorname{Tr} \bar{S}_{\pi}(I-P)+4 \operatorname{Tr} S_{0}\left(I-P_{0}\right)+4(M-1) \mathrm{e}^{-[\Delta H(\pi)-2 \delta T]} \\
& \leqslant \frac{8 \sum_{j \in I_{T}} F\left(N_{j}+m_{j}^{*}\right)}{\delta^{2} T^{2}}+\frac{4 \sum_{j \in I_{T}} F\left(N_{j}\right)}{\delta^{2} T^{2}}+4(M-1) \mathrm{e}^{-\left[C_{T}-2 \delta T\right]} .
\end{aligned}
$$

Поскольку функция $F(x)$ ограничена, а количество элементов множества $I_{T}$ пропорционально $T$, суммы в числителях имеют порядок $T$, и первые два слагаемых имеют порядок $T^{-1}$. Для завершения доказательства остается показать, что

$$
\liminf _{T \rightarrow \infty} \frac{C_{T}}{T} \geqslant C
$$

Пусть функция $m^{*}(\omega)$ дается соотношением $(96)$, и пусть $\omega_{j}^{\prime}$ - точка отрезка $\left[\omega_{j-1}, \omega_{j}\right]$, в которой эта функция достигает минимума, тогда

$$
\frac{1}{2 \pi} \sum_{j \in I_{T}} \hbar \omega_{j}^{\prime} m^{*}\left(\omega_{j}^{\prime}\right) \leqslant \frac{1}{2 \pi} \int_{\underline{\omega}}^{\bar{\omega}} \hbar \omega m^{*}(\omega) d \omega=E,
$$


откуда следует, что

$$
\frac{C_{T}}{T} \geqslant \frac{1}{2 \pi} \sum_{j \in I_{T}}\left[g\left(N\left(\omega_{j}\right)+m^{*}\left(\omega_{j}^{\prime}\right)\right)-g\left(N\left(\omega_{j}\right)\right)\right] \Delta \omega_{j} .
$$

Поскольку функции $N(\omega)$ и $m^{*}(\omega)$ непрерьвны, суммы в правой части стремятся к

$$
\int_{\underline{\omega}}^{\bar{\omega}}\left[g\left(N(\omega)+m^{*}(\omega)\right)-g(N(\omega))\right] d \omega=C,
$$

и доказательство завершено.

Обратимся теперь к случаю неограниченного диапазона частот $(0, \infty)$. Рассуждения, аналогичные проведенньм выше, показьвают, что достаточно установить соотношение

$$
\lim _{T \rightarrow \infty} \frac{C_{T}}{T}=C(0, \infty) \equiv \max _{\mathscr{M}(0, \infty)} \frac{1}{2 \pi} \int_{0}^{\infty}[g(N(\omega)+m(\omega))-g(N(\omega))] d \omega
$$

где

$$
\mathscr{M}(0, \infty)=\left\{m(\cdot): m(\omega) \geqslant 0, \frac{1}{2 \pi} \int_{0}^{\infty} \hbar \omega m(\omega) d \omega \leqslant E\right\}
$$

Максимум достигается на функции $m^{*}(\omega)$ вида $(96)$, где $\theta$ выбирается так, что

$$
\frac{1}{2 \pi} \int_{0}^{\infty} \hbar \omega m^{*}(\omega) d \omega=E .
$$

Выберем числа $0<\underline{\omega}<\bar{\omega}<\infty$. Опуская частоты, лежашие вне этого диапазона, получаем

$$
\begin{aligned}
& \liminf _{T \rightarrow \infty} \frac{C_{T}}{T} \geqslant \max _{\underline{\mathcal{M}}(\underline{\omega}, \bar{\omega})} \frac{1}{2 \pi} \int_{\underline{\omega}}^{\bar{\omega}}[g(N(\omega)+m(\omega))-g(N(\omega))] d \omega \\
& \geqslant \frac{1}{2 \pi} \int_{\underline{\omega}}^{\bar{\omega}}\left[g\left(N(\omega)+m^{*}(\omega)\right)-g(N(\omega))\right] d \omega,
\end{aligned}
$$

поскольку $m^{*}(\cdot) \in \mathscr{M}(\underline{\omega}, \bar{\omega})$. Переходя к пределу при $\underline{\omega} \rightarrow 0, \bar{\omega} \rightarrow \infty$, получаем знак $\geqslant$ в соотношении (107).

Чтобы доказать обратное неравенство, рассмотрим соотношение

$$
\frac{C_{T}}{T}=\frac{1}{2 \pi} \sum_{j}\left[g\left(N_{j}+m_{j}^{*}\right)-g\left(N_{j}\right)\right] \Delta \omega_{j},
$$

где

$$
m_{j}^{*}=\left(\frac{1}{\mathrm{e}^{\theta_{T} \hbar \omega_{j}}-1}-N_{j}\right)_{+}
$$


и $\theta_{T}$ выбрано таким образом, что

$$
\frac{1}{2 \pi} \sum_{j} \hbar \omega_{j} m_{j}^{*} \Delta \omega_{j}=E .
$$

Вводя кусочно-постоянные функции

$$
N_{T}(\omega)=N_{j}, \quad m_{T}(\omega)=m_{j}^{*} \text { при } \omega_{j-1}<\omega \leqslant \omega_{j},
$$

мы можем переписать правую часть соотношения (109) в виде

$$
\begin{aligned}
\frac{1}{2 \pi} & \int_{0}^{\infty}\left[g\left(N_{T}(\omega)+m_{T}(\omega)\right)-g\left(N_{T}(\omega)\right)\right] d \omega \\
& =\frac{1}{2 \pi} \int_{0}^{\infty}\left[g\left(N(\omega)+m_{T}(\omega)\right)-g(N(\omega))\right] d \omega \\
& +\frac{1}{2 \pi} \int_{0}^{\infty}\left[g\left(N_{T}(\omega)+m_{T}(\omega)\right)-g\left(N(\omega)+m_{T}(\omega)\right)+g(N(\omega))-g\left(N_{T}(\omega)\right)\right] d \omega .
\end{aligned}
$$

Принимая во внимание тот факт, что

$$
\frac{1}{2 \pi} \int_{0}^{\infty} \hbar \omega m_{T}(\omega) d \omega \leqslant \frac{1}{2 \pi} \sum_{j} \hbar \omega_{j} m_{j}^{*} \Delta \omega_{j}=E
$$

получаем, что первое слагаемое менњше или равно

$$
\max _{\mathscr{M}(0, \infty)} \frac{1}{2 \pi} \int_{0}^{\infty}[g(N(\omega)+m(\omega))-g(N(\omega))] d \omega=C(0, \infty) .
$$

Остается показать, что второе слагаемое стремится к нулю. Это будет следовать из теоремы Лебега о доминированной сходимости. Поскольку $N(\omega)$ непрерывна, $N_{T}(\omega) \rightarrow N(\omega)$ и $g\left(N_{T}(\omega)\right) \rightarrow g(N(\omega))$ поточечно. Далее, заметим, что $\theta_{T}$ отделены от нуля, когда $T \rightarrow \infty$. В самом деле, если $\theta_{T} \downarrow 0$ для некоторой последовательности $T \rightarrow \infty$, то соответствуюшая последовательность непрерывных функций

$$
\left(\frac{1}{\mathrm{e}^{\theta_{T} \hbar \omega}-1}-N(\omega)\right)_{+}
$$

стремится к $\infty$ равномерно на любом интервале $0<\underline{\omega} \leqslant \omega \leqslant \bar{\omega}<\infty$, что противоречит условию (110). Отсюда следует, что для любого $\omega>0$ величины

$$
N(\omega)+m_{T}(\omega)=\max \left(\frac{1}{\mathrm{e}^{\theta_{T} \hbar \omega}-1}, N(\omega)\right)
$$

остаются ограниченными при $T \rightarrow \infty$. Поскольку функция $g(\omega)$ равномерно непрерьвна на любом отрезке, имеем

$$
g\left(N_{T}(\omega)+m_{T}(\omega)\right)-g\left(N(\omega)+m_{T}(\omega)\right) \rightarrow 0
$$


Остается показать, что подынтегральные выражения ограничены интегрируемой функцией. Принимая во внимание тот факт, что $h^{\prime \prime}(x) \leqslant 0$ при $x \geqslant 0$, получаем $g(x+y)-g(x) \leqslant g(y)$ при $x, y \geqslant 0$. Поэтому подьнтегральное выражение ограничено функцией $2 g\left(m_{T}(\omega)\right)$. Имеем

$$
m_{T}(\omega) \leqslant \frac{1}{\mathrm{e}^{\theta_{T} \hbar \omega}-1} \leqslant \frac{1}{\mathrm{e}^{\theta_{0} \hbar \omega}-1} .
$$

Таким образом,

$$
g\left(m_{T}(\omega)\right) \leqslant g\left(\frac{1}{\mathrm{e}^{\theta_{0} \hbar \omega}-1}\right)=\frac{\theta_{0} \hbar \omega}{\mathrm{e}^{\theta_{0} \hbar \omega}-1}-\log \left(1-\mathrm{e}^{-\theta_{0} \hbar \omega}\right),
$$

где правая часть является положительной интегрируемой функцией.

Формулы для пропускной способности имеют особенно простой вид в случае равновесного шума $N(\omega)=N_{\theta_{P}}(\omega) \equiv\left(\mathrm{e}^{\theta_{P} \hbar \omega}-1\right)^{-1}$, где $\theta_{P}$ определяется из уравнения

$$
\frac{1}{2 \pi} \int_{0}^{\infty} \frac{\hbar \omega}{\mathrm{e}^{\theta_{P} \hbar \omega}-1} d \omega=P
$$

Используя формулу

$$
\int_{0}^{\infty} \frac{x}{\mathrm{e}^{x}-1} d x=\frac{\pi^{2}}{6}
$$

получаем $\theta_{P}=\sqrt{\pi /(12 \hbar P)}$, и

(111) $\frac{1}{2 \pi} \int_{0}^{\infty} g\left(N_{\theta_{P}}(\omega)\right) d \omega=\frac{1}{2 \pi} \int_{0}^{\infty}\left\{\frac{\theta_{P} \hbar \omega}{\mathrm{e}^{\theta_{P} \hbar \omega}-1}-\log \left(1-\mathrm{e}^{-\theta_{P} \hbar \omega}\right)\right\} d \omega=\sqrt{\frac{\pi P}{3 \hbar}}$, откуда

$$
C(0, \infty)=\frac{1}{2 \pi} \int_{0}^{\infty}\left[g\left(N_{\theta_{P+E}}(\omega)\right)-g\left(N_{\theta_{P}}(\omega)\right)\right] d \omega=\sqrt{\frac{\pi(P+E)}{3 \hbar}}-\sqrt{\frac{\pi P}{3 \hbar}}
$$

что совпадает с пропускной способностью фотонного канала с неограниченным диапазоном частот, вычисленной в работе [34].

Попытаемся теперь дать формулировку задачи в терминах предельного случайного процесса. В пределе $T \rightarrow \infty$ следует ожидать, что периодический процесс (79) переходит в

$$
X(t)=\alpha(t)+Y(t), \quad t \geqslant 0,
$$

где $\alpha(t)$ - классический сигнал, а $Y(t)$ - квантовый гауссовский шум

$$
Y(t)=\int_{0}^{\infty} \sqrt{\hbar \omega}\left(d A_{\omega} \mathrm{e}^{-i \omega t}+d A_{\omega}^{\dagger} \mathrm{e}^{i \omega t}\right) .
$$


Здесь $A_{\omega}, \omega \geqslant 0,-$ квантовый гауссовский процесс с независимыми приращениями, характеризуюшийся коммутатором

$$
\left[d A_{\omega}, d A_{\lambda}^{\dagger}\right]=\delta(\omega-\lambda) d \omega d \lambda,
$$

с нулевым средним и корреляционной функцией

$$
\left\langle d A_{\omega}^{\dagger} d A_{\lambda}\right\rangle=\delta(\omega-\lambda) N(\omega) d \omega d \lambda
$$

Подставляя в (113), получаем коммутатор

$$
[Y(t), Y(s)]=2 i \hbar \int_{0}^{\infty} \omega \sin \omega(s-t) d \omega=2 i \hbar \pi \delta^{\prime}(t-s)
$$

и корреляционную функцию квантового шума в виде

$$
\langle Y(t) Y(s)\rangle=B(t-s)+K(t-s),
$$

где

$$
B(t)=2 \hbar \int_{0}^{\infty} \omega N(\omega) \cos \omega t d \omega
$$

a

$$
K(t)=\hbar \int_{0}^{\infty} \omega \mathrm{e}^{-i \omega t} d \omega=-\hbar\left[t^{-2}-i \pi \delta^{\prime}(t)\right]
$$

- корреляционная функция вакуумного шума (отвечаюшая нулевой температуре).

Процесс $X(t)$ наблюдается на временном отрезке $[0, T]$. Это означает, что рассматриваются гауссовские (квазисвободные) состояния со средними $\alpha(t)$ и коррелящионой функцией $(115)$, на $C^{*}$-алгебре канонических коммутационных соотношений, порождаемой операторами $X(t) ; t \in[0, T]$, и определяемой коммутатором (114) (см., например, [21]). Пропускная способность при ограничении (87) на мошность сигнала определяется так же, как непосредственно перед формулировкой теоремы 5. Доказательство этой теоремы делает правдоподобным утверждение, что пропускная способность такого канала дается выражением (112). Однако классический метод доказательства сведением к параллельньм каналам с одной степенью свободы [12] встречает здесь новые трудности. Во-первых, ядра (114), (115) являются уже обобшенньми функциями. Более существенно то обстоятельство, что в классическом случае имеются лишь две квадратичные формы, задаваемые корреляционной функцией и энергетическим ограничением (последняя есть просто скалярное произведение в $L^{2}$ ), которые могут быть одновремено диагонализованы путем решения интегрального уравнения с ядром (115). В квантовом случае добавляется кососимметричная форма, определяемая коммутатором, которая также должна быть приведена к каноническому виду, для получения разложения на независимые квантовые степени свободы. Это, однако, невозможно сделать при конечном $T$, хотя, как показьвает доказательство теоремы 5 , это происходит в пределе (при $T \rightarrow \infty$ ). 


\section{СПИСОК ЛИТЕРАТУРЫ}

[1] Barnum H., Knill E., Nielsen M. A. On quantum fidelities and channel capacities // LANL $^{1}$ Report no. quant-ph/9809010, Sep. 1998.

[2] Barnum H., Nielsen M. A., Schumacher B. Information transmission through noisy quantum channels // LANL Report no. quant-ph/9702049, Feb. 1997. See also Phys. Rev. A. 1998. V. 57, P. 4153-4175.

[3] Bennett C. H., Fuchs C. A., Smolin J. A. Entanglement-enhanced classical communication on a noisy quantum channel // Quantum Communication, Computing and Measurement, Proc. QCM96 / ed. O. Hirota, A.S. Holevo, C.M. Caves. New York: Plenum, 1997. P. $79-88$.

[4] Bennett C.H. Classical and quantum information transmission and interactions // Quantum Communication, Computing and Measurement, Proc. QCM96 / ed. O. Hirota, A. S. Holevo, C.M. Caves. New York: Plenum, 1997. P. 25-39.

[5] Бурнашев М.В., Холево А.С. О функции надежности квантового канала связи // LANL Report no. quant-ph/9703013, March 1997.

[6] Calderbank A.R., Shor P.W. Good quantum error-correcting codes exist // Phys. Rev. A. 1996. V. 54. P. 1098-1105.

[7] Caves C.M., Drummond P.B. Quantum limits of bosonic communication rates // Rev. Modern Phys. 1994. V. 66. № 2. P. 481-538.

[8] Cover T. M., Thomas J. A. Elements of Information Theory. New York: Wiley, 1991.

[9] Echigo M., Nakamura M. A remark on the concept of channels // Proc. Japan Acad. 1962. V. 38. № 5. P. 307-309.

[10] Forney G.D., Jr. S. M. Thesis. Cambridge, MA: MIT, 1963.

[11] Fujivara A., Nagaoka H. Operational capacity and semi-classicality of quantum channel // IEEE Trans. Inform. Theory. 1998. V. 44. P. 1071-1086.

[12] Галлагер Р. Теория информации и надежная связь. М.: Сов. Радио, 1974.

[13] Gordon J.P. Quantum effects in communication systems // Proc. IRE. 1962. V. 50. № 9. P. 1898-1908.

[14] Gordon J. P. Noise at optical frequencies; information theory // Quantum Electronics and Coherent Light, Proc. Int. School Phys. "Enrico Fermi", Course XXXI / ed. P. A. Miles. New York: Academic Press, 1964. P. 156-181.

[15] Hausladen P., Jozsa R., Schumacher B., Westmoreland M., Wootters W. Classical information capacity of a quantum channel // Phys. Rev. A. 1996. V. 54. № 3. P. $1869-1876$.

[16] Хелстром К. Квантовая теория проверки гипотез и оценивания. М.: Мир, 1978.

[17] Хинчин А.Я. Об основных теоремах теории информации // УМН. 1956. Т. 11. № 1. C. $17-75$.

[18] Холево А. С. К математической теории квантовых каналов связи // Проблемы передачи информации. 1972. Т. 8. № 1. С. 63-71.

[19] Холево А. С. Некоторые оценки количества информации, передаваемого квантовым каналом связи // Проблемы передачи информации. 1973. Т. 9. № 3. С. 3-11.

[20] Холево А. С. Замечания об оптимальных квантовых измерениях // Проблемы передачи информации. 1974. Т. 10. № 4. С. 51-55.

[21] Холево А.С. Исследования по общей теории статистических решений // Тр. МИАН. 1976. T. 124

[22] Holevo A.S. Problems in the mathematical theory of quantum communication channels // Rep. Math. Phys. 1977. V. 12. № 2. P. 273-278.

[23] Холево А. С. Об асимптотически оптимальной проверке гипотез в математической статистике // Теория вероятн. и ее примен. 1978. Т. 23. № 2. С. 429-432.

[24] Холево А. С. О пропускной способности квантового канала связи // Проблемы передачи информации. 1979. Т. 15. № 4. С. 3-11.

\footnotetext{
${ }^{1}$ Адрес электронного архива LANL (Лос-Аламосская Национальная Лаборатория США): http://xxx.lanl.gov/archive/
} 
[25] Холево А. С. Вероятностные и статистические аспекты квантовой теории. М.: Наука, 1980.

[26] Holevo A.S. The capacity of quantum communication channel with general signal states // LANL Report no. quant-ph/9611023, Nov. 1996. See also IEEE Trans. Inform. Theory. 1998. V. 44, № 1, P. 269-272.

[27] Holevo A.S. On quantum communication channels with constrained inputs // LANL Report no. quant-ph/9705054, May 1997.

[28] Holevo A.S. Coding theorems for quantum channels // Tamagawa University Research Review. 1998. № 4 .

[29] Jozsa R., Schumacher B. A new proof of the quantum noiseless coding theorem // J. Modern Optics. 1994. V. 41. № 12. P. 2343-2349.

[30] Kato K., Osaki M., Suzuki T., Ban M., Hirota O. Upper bound of the accessible information and lower bound of the Bayes cost in quantum signal detection processes // Quantum Communication, Computing and Measurement, Proc. QCM96 / ed. O. Hirota, A. S. Holevo, C. M. Caves. New York: Plenum, 1997. P. 63-71.

[31] Kossakowski A. Talk at the Symposium on Quantum Probability and Applications, Gdańs, 1997 (unpublished).

[32] Kraus K. States, Effects and Operations. Fundamental notions of quantum theory. Lecture Notes in Physics. V. 90. Berlin: Springer-Verlag, 1983.

[33] Крейн М.Г. О базисах Бари пространства Гильберта // УМН. 1957. Т. 12.№3. C. $337-341$.

[34] Лебедев Д. С., Левитин Л.Б. Максимальное количество информации, передаваемое квантовым каналом связи // Докл. АН СССР. 1963. Т. 149. С. 1299-1303.

[35] Левитин Л.Б. О квантовой мере информации // Tруды IV Всесоюзного совещания по передаче информации и теории кодирования. Ташкент, 1969. С. 111-115.

[36] Levitin L.B. Optimal quantum measurement for two pure and mixed states // Quantum Communications and Measurement, Proc. QCM94 / ed. V.P. Belavkin, O. Hirota, R. L. Hudson. New York: Plenum, 1995. P. 439-448.

[37] Lieb E.H., Ruskai M. B. Proof of the strong subadditivity of quantum mechanical entropy // J. Math. Phys. 1973. V. 14. P. 1938-1941.

[38] Lindblad G. Entropy, information and quantum measurements // Commun. Math. Phys. 1973. V. 33. P. 305-322; Expectations and entropy inequalities for finite quantum systems 1974. V. 39. P. 111-119; Completely positive maps and entropy inequalities 1975 . V. 40. P. $147-151$.

[39] Наймарк М.А. Спектральные функции симметричного оператора // Изв. АН СССР. Сер. матем. 1940. Т. 4. № 3. C. 277-318.

[40] Ohya M., Petz D. Quantum Entropy and Its Use. Berlin: Springer-Verlag, 1993.

[41] Sasaki M., Kato K., Izutsu M., Hirota O. Quantum channels showing superadditivity in the capacity // Preprint: Tamagawa University, 1997.

[42] Schumacher B., Westmoreland M. D. Sending classical information via noisy quantum channel // Phys. Rev. A. 1997. V. 56. № 1. P. 131-138.

[43] Steane A. Quantum computing // LANL Report no. quant-ph/9708022, Sep. 1997.

[44] Stinespring W. F. Positive functions on $C^{*}$-algebras // Proc. Amer. Math. Soc. 1955. V. 6. P. 211-216.

[45] Stratonovich R. L., Vantsyan A. G. Asymptotically error-free decoding in pure quantum channels // Probl. Control Inform. Theory. 1978. V. 7. №3. P. 161-174.

[46] Uhlmann A. Relative entropy and the Wigner-Yanase-Dyson-Lieb concavity in an interpolation theory // Commun. Math. Phys. 1977. V. 54. P. 21-32.

[47] Wehrl A. General properties of entropy // Rev. Mod. Phys. 1978. V. 50. № 2. P. 221-260.

[48] Yuen H.P., Ozawa M. Ultimate information carrying limit of quantum systems // Phys. Rev. Lett. 1993. V. 70. № 4. P. 363-366. 